\title{
Dynamically structured fluidization: Oscillating the gas flow and other opportunities to intensify gas-solid fluidized bed operation
}

\author{
Victor Francia, Kaiqiao Wu, Marc-Olivier Coppens ${ }^{*}$ \\ EPSRC “Frontier Engineering” Centre for Nature Inspired Engineering, Department of Chemical Engineering, University \\ College London, Torrington Place, London WC1E 7JE, United Kingdom,E-mail: 느.coppens@ucl.ac.uk
}

Various approaches to structure gas-solid fluidized beds are reviewed, followed by detailed discussion on the use of gas pulsation to induce dynamic structuring. Granular media are dissipative systems, which develop complex spatiotemporal patterns when excited by an oscillating energy source. Here, we discuss how such perturbations initiate surface patterns and how these could propagate into a macroscopically organized flow. We call this dynamically structured fluidization. Vibrated shallow granular layers form ordered surface waves. The hydrodynamics of pulsed gas-fluidized layers are related, but more complex: Under appropriate conditions, surface waves transition into a threedimensionally ordered bubbling flow. This occurs in much deeper granular beds than under vibration, indicating distinct physics. In this dynamically structured state, bubbles organize into a scalable subharmonic, triangular lattice that is highly predictable and responsive to changes in oscillation parameters, allowing for an unprecedented level of control. Structured bubbling is observed only under sufficiently dense conditions; thus, a dynamically structured fluidized bed sits between fixed and fluidized beds, offering opportunities for process intensification, due to less macromixing than traditional fluidization, but a higher level of control through micromixing. This informs new intensified designs for processes that are highly exothermic, involve particle formation, thermally sensitive or high-value materials.

Keywords: fluidization; intensification; oscillatory flow; structuring; pulsation; dynamics

\section{Intensification, structuring and scale-up of fluidized beds}

Fluidization excels as a way to put fluids and solids in intense contact. It is used in applications where homogeneous mixing, heat and mass transfer are important, such as dryers or multiphase reactors, e.g., for gasification, polymerization 
or fluid catalytic cracking (FCC) [1]. Despite widespread application, many aspects of the operation and scale-up of fluidized beds are still troublesome [2, 3]. Most materials are hard to fluidize uniformly. Coarse particles receive too little energy, due to their low surface area to volume ratio. Fine particles are too cohesive, and in many practical scenarios flowability is hindered by the presence of liquid binders. The rheology of a gas-solid suspension is affected by a broad range of interparticle forces [4] that are hard to quantify and are highly dependent on materials and process conditions [5]. Furthermore, its hydrodynamics are inherently unstable [6]. Mixing is caused by flow structures covering a wide range of spatial and temporal scales and, unlike single-fluid turbulent flow, the interplay of hydrodynamic and interparticle forces creates a variety of complex flow features, which are very challenging to predict using current computational tools. Most are scale-dependent, and the behavior of a lab-scale unit can differ drastically from a pilot unit, let alone a full-scale industrial bed [7-9]. Given this level of complexity, it has been common to regard a gas-solid fluidized bed as an idealized suspension under an instability $[10,11]$ or to describe it as a deterministic chaotic system, in which a small disturbance, such as a fluctuation in upstream gas flow or packing, can degenerate into completely different hydrodynamics $[12,13]$. This makes reliable scale-up and robust control of real units very difficult.

The performance of a bed depends on the contact pattern between gaseous and solid species [14]. Mixing and residence time vary drastically between different designs, raw materials and scales. In a suspension of Geldart B particles, the solid motion is affected by the formation of bubbles. A rising bubble conveys the solids up and towards the center, after which solids recirculate downward near the walls (Figure 1a) [15]. This improves gas-solid contact, because the solids mix inside the bubble wakes (Figure 1b), but, while large bubbles create mixing, they also allow part of the gas to bypass the solid phase, thus reducing the time available for reaction or interfacial transport. Designing a fluidized bed involves compromises between vigorous mixing and sufficient contact time, by optimizing bubble size and velocity [16]. While the bubble dynamics are scale-dependent and hard to predict [17], there are various ways to alter the overall hydrodynamics to improve particular processes $[18,19]$. These include altering the interparticle forces by adding flowconditioning particles [20,21], liquid binders [22, 23], adsorption of gas and changes to the operating conditions [24, 25]. When possible, fluid viscosity and density could be modified to alter the flow regime by adequate selection of the gas [26], temperature and pressure [27, 28], and even working under supercritical conditions, common in the pharmaceutical $[29,30]$ and energy sectors $[31,32]$. There are also many ways to supply an additional source of energy in so-called "assisted fluidization" technologies, for example with direct solar irradiation [33, 34], using microwaves in fast biomass pyrolysis [35], or introducing radio frequency [36, 37] and ultrasound [38, 39] in the design of fluidized bed dryers. 
Such changes to materials, process conditions or environment can drastically increase gas-solid contact, for example, by reducing bubble size or stopping a cohesive powder from channelling, and thus improve performance and reduce uncertainty in fluidized bed design and scale-up. However, rather than a fundamentally new design, they offer a range of ad hoc, often empirical solutions to improve the efficiency of specific processes.

Alternatively, one can try to overcome the disadvantages of traditional fluidization with more fundamental design changes. Instead of allowing the hydrodynamic instability to degenerate into uncontrolled chaos, one can try to suppress $\mathrm{r}$ contain this degeneration. Here, one seeks to modify key aspects of the design, using additional degrees of freedom to create a "structured fluidized bed" where the flow has a much higher degree of predictability or is, at least, more homogeneous and scalable. The intention is to either change an intrinsic property of the system or apply an external
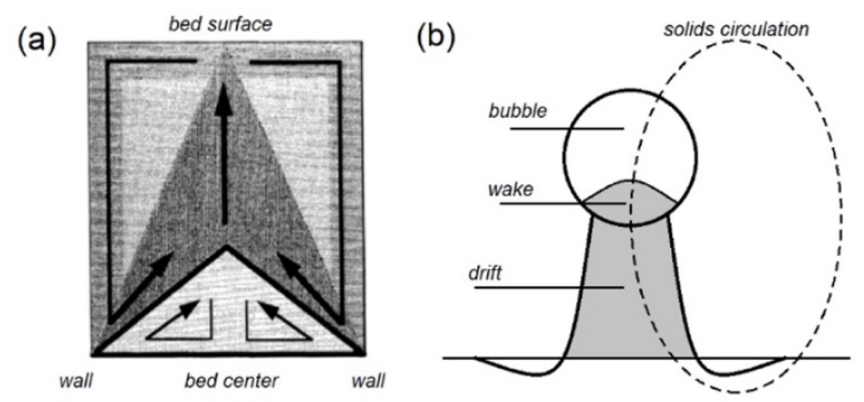

Figure 1: Solid mixing mechanisms in bubbling fluidized beds. (a) long-range, (b) local circulation. Reproduced with permission [15]

o actuation to stabilize the flow. This improves scalability by decoupling conflicting design objectives, such as mixing versus residence time. Using external actuation also equips us with a new set of tools or degrees of freedom to manipulate the operation of the bed and tailor transport rates, reaction conversion or selectivity, not only improving performance but also enabling better control and process integration. A decade ago, van Ommen et al. [40] summarized routes to design a structured gas-solid fluidized bed. Table 1 and Figure 2 update this classification and list approaches according to whether they impose a dynamic or a geometrical constraint in either phase, and to how they apply an additional source of energy to the bed, namely through a contact (particle-particle, particle-wall) force, a surface (particle-fluid e.g. drag, pressure) force or body/volume forces (electromagnetic, gravity). Figure 3 lists some examples of structured devices.

One can introduce geometrical constraints into the solids themselves or alter their environment. Polydispersity, shape and roughness are examples of particle properties that alter both interparticle forces and fluid-particle forces [41] in a way that is not fully predictable. A clear expression of this are the known discrepancies between the many correlations to predict the minimum fluidization velocity of different powders [42]. Many works now focus on accounting for the 
effect of the particles' non-sphericity [43] and develop correlations suited to polydisperse systems [44, 45], not only at ambient conditions, but also at high temperatures and pressures [46]. However, it is not always possible to control the specifications of raw materials, and the effect of changing particle properties is neither localized nor understood well enough to utilize them in general to impose a well-controlled, macroscopic flow pattern. Still, this method is used empirically: Changes in particle size can improve flowability, reduce attrition or overall mass transfer limitations. For example, Sun and Grace demonstrated that broad catalyst size distributions can pack densely and improve conversion by reducing the bubble size [47], while Brouwer et al. [21] show the correlation between the content in fines and the reduction in bubble size in a bed of Geldart A alumina particles. Fines can be added to homogenize a bed by filling interstitial gaps (hydrodynamics) and reducing attrition (contact forces) [48]; coarse particles can also improve the flowability of fine cohesive powders by breaking up agglomerates [49].

Changing the enclosure in which the solids move is a more versatile way to alter geometry. The use of internals, such as baffles, distributors, tubes or membranes, allows an engineer to redistribute the gas flow and prevent bubble growth

Table 1: Summary of methods to structure fluidized beds *

Force

* Two token references are given for each case; more are discussed in the main body of the text. 

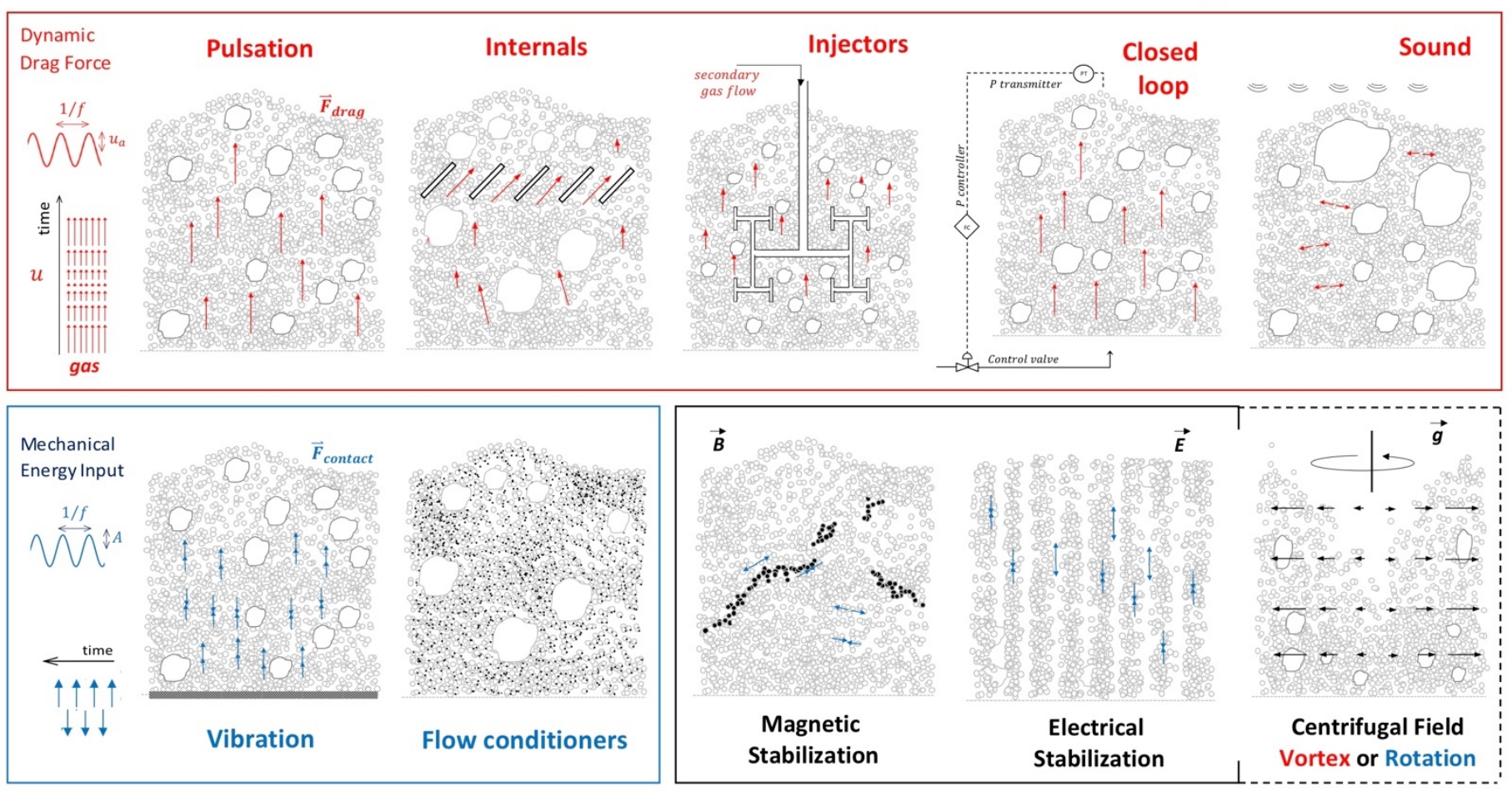

(b) Particle-Particle Contact Forces

(c) Body (or Pseudo-Body) Forces

Figure 2: Depiction of methods to structure a fluidized bed by changing (a) particle-fluid forces: pulsation, internals and injectors, closed-loop control of gas flow, use of (pressure) sound waves, or a complex vortex flow; (b) particle-particle or particle-wall contact forces under mechanical vibration, via particle properties (flow conditioners) or moving boundaries in rotating fluidized beds (RFB); or (c) the action of an external electromagnetic field.

[71]. Although systematic approaches have been put forward, in general, internals are designed ad hoc, for each particular technology and even patented for "unanticipated improvements" for different devices. Zhang et al. [72] studied the effect of louver baffles on bubbling hydrodynamics of FCC catalyst, concluding that the baffles can break up bubbles and improve gas hold-up, but also create undesired secondary flow patterns. Yang et al. studied their effect computationally in 3D geometries, capturing the distribution of solids and the suppression of axial mixing [73]. Arrays of vertical elements in the form of heat exchange tubes and membranes are very often present in gas-solid reactors. Maurer et al. described their effect on the hydrodynamics of a cold flow column using X-ray tomography [53]. They observed that arrayed vertical tubes improve the horizontal distribution of bubbles, leading to a $60 \%$ reduction in hydraulic bubble volume and a narrower size distribution in a mixture of Geldart A and B $\gamma$-alumina particles. More recently, experimental studies confirm the improvement in the heat transfer efficiency for beds of Geldart B particles [74]. Of course, the use of internals introduces a trade-off between better bubbling and an overall higher energy requirement. For example, Yang et al. conducted experiments with a series of perforated plates [54] that were shown to 
suppress back-mixing in a bed of Geldart A particles, but at the cost of elevated pressure drop and substantial extra energy dissipation.

Instead of using obstacles to direct the gas, one can use multiple injection points or a 3D gas injector to alter how the particle-fluid forces are distributed over the bed. In a bed, one can distinguish a primary gas flow that is, at least, required to maintain the particles fluidized, from a secondary one used to increase the gas feed and mix the suspension. In a traditional bubbling unit, the secondary flow enters through the bottom and forms bubbles, for Geldart B particles, that degenerate into a chaotic system as they move up, break and coalesce. One cannot avoid this instability in the limit, but it is possible to keep the system removed from equilibrium and delay the development of instabilities, by using additional inlets to distribute the secondary gas through the entire volume of the bed. At each injection point, a small fraction of the secondary gas flow leads to localized micromixing. This creates smaller bubbles, and the separation of the inlets reduces their interaction. Every inlet creates a degree of mixing related to the local gas flow rate, but also to the spatial distribution of the inlets and whether their zones of hydrodynamic influence interact. Judicious placement of the gas inlets in the bed provides a new degree of freedom to control mixing, decoupling it from the chaotic evolution when all bubbles emerge from the bottom. For scalability, it is important that the local conditions around each outlet are similar or independent of each other. To design an optimal structure, one can look into how natural systems, like the vascular network, trees and lungs have evolved to optimize transport in 3D in a scalable way through fractal growth [75]. Delivering the secondary flow through a set of outlets at the end of a fractal tree ensures a homogeneous distribution (Figure 3c). This structure is able to self-regulate, so that each outlet receives the same flow rate and backpressure, and thanks to its self-similar nature, it can be scaled up, maintaining the same local environment at every gas outlet to the injector, or inlet to the bed, irrespective of scale. Nevertheless, a fluidized bed is an inherently anisotropic system, due to the action of gravity, pressure drop or axial depletion of reactants, so one must expect a certain level of interaction between mixing cells in vertical direction. A fractal dimension smaller than 3 (volume filling - introducing the flow through points distributed over the entire bed volume) but larger than 2 (area filling - typically, introducing the flow through the bottom) is thus expected to be optimal for beds of different scales, using the length of the final fractal generation as the scale up parameter [76,77]. Christensen et al. studied the impact of a fractal injector in a fluidized bed reactor and noted improved performance in terms of conversion and selectivity; this was associated to better micromixing, homogenization of the radial gas distribution, an abrupt reduction in bubble size, and overall hydrodynamics approaching plug flow behavior with fresh fluid distribution at various levels vertically [57, 58]. 
Internals and injectors are effective ways to direct the gas flow, but they can be intrusive and are typically fixed solutions, once in place. Some type of external actuation offers more flexibility to new design concepts. One such option is to introduce a pseudo-body force to alter the gravitational pull. So-called "high-gravity fluidized beds" impart rotation to the solids and use this new source of inertia to control how and where particles concentrate. A vortex gas flow realizes this by transmitting the energy in the tangential motion of a swirling gas flow to the solids through drag. Using the swirl to concentrate the solids allows one to change drastically the pressure drop, the minimum fluidization velocity and the elutriation rate [78].

Vortex flows have attracted interest in fluidization research for a long time [61]. They underpin swirl fluidized beds and combustors $[79,80]$, and various designs of vortex chambers for catalytic reactions and biomass processing, e.g., rotating fluidized beds in static geometry, gas-solid vortex reactors (GSVR) and Torbed ${ }^{\circledR}$ reactors [81-83]. As for other cyclonic flows in separators, reverse cyclones and spray dryers, the intensity of a vortex could be potentially modulated as an additional degree of freedom to change mixing, contact patterns and even the stresses sustained by the solids as they concentrate. This is relevant for the fluidization of cohesive powders and to control final particle properties in product-driven operations, such as coating [84]. The swirl is typically imparted to the gas with a blade injector [85], tangential inlets $[86,87]$, or a combination of both, but similar effects can be achieved with a modified distributor [88, 89]. One can also impose the rotation using a moving boundary in so-called rotating, rotational or centrifugal fluidized beds. In this case, the solids and the gas phase acquire centrifugal inertia as a result of friction with the walls. For example, Quevedo et al. [68] studied the influence of centrifugal fluidization on nanoparticles. Rotating the unit at accelerations from 0 to $40 \mathrm{~g}$ allowed them to increase the bed pressure drop and the minimum fluidization velocity, so that much larger gas velocities could be used without entrainment. Other ways to introduce a body force rely on an external electromagnetic force field. The use of electrical and magnetic actuation has been studied for many years [63, 92]. The presence of a magnetic field increases interparticle forces by creating particle chains by dipole-dipole interactions that alter the hydrodynamics. For more than two decades, Hristov has set out a systematic approach to magnetically assisted fluidization [93]. Comprehensive experimentation has shown how magnetic forces change the rheology of fluidized powders, including how they can extend the homogeneous expansion of Geldart A powders [94], and stabilize bubbling in Geldart B particles [95]. Dense, jammed beds are not responsive, because particles need to be free enough to restructure upon actuation and, as a result, changes to the rheology under aeration also depend on particle size, polydispersity and the bed microstructure [96]. Recent studies have looked into the effects of the orientation of the magnetic field versus the gas direction [97] and a material-dependent effect of temperature in reducing the apparent 
cohesiveness of the powder [98]. Magnetic stabilization of a fluidized bed has led to the development of catalysts [99] and many new types of fluidized bed reactors, particularly for L-S or G-L-S systems [100, 101]. The imposition of a
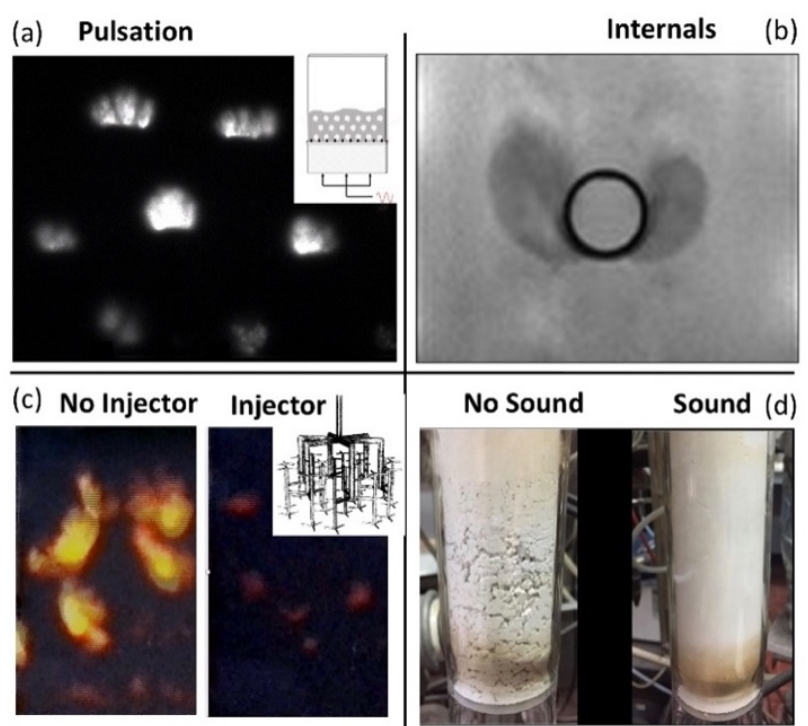

(e) Vibration \& Pulsation
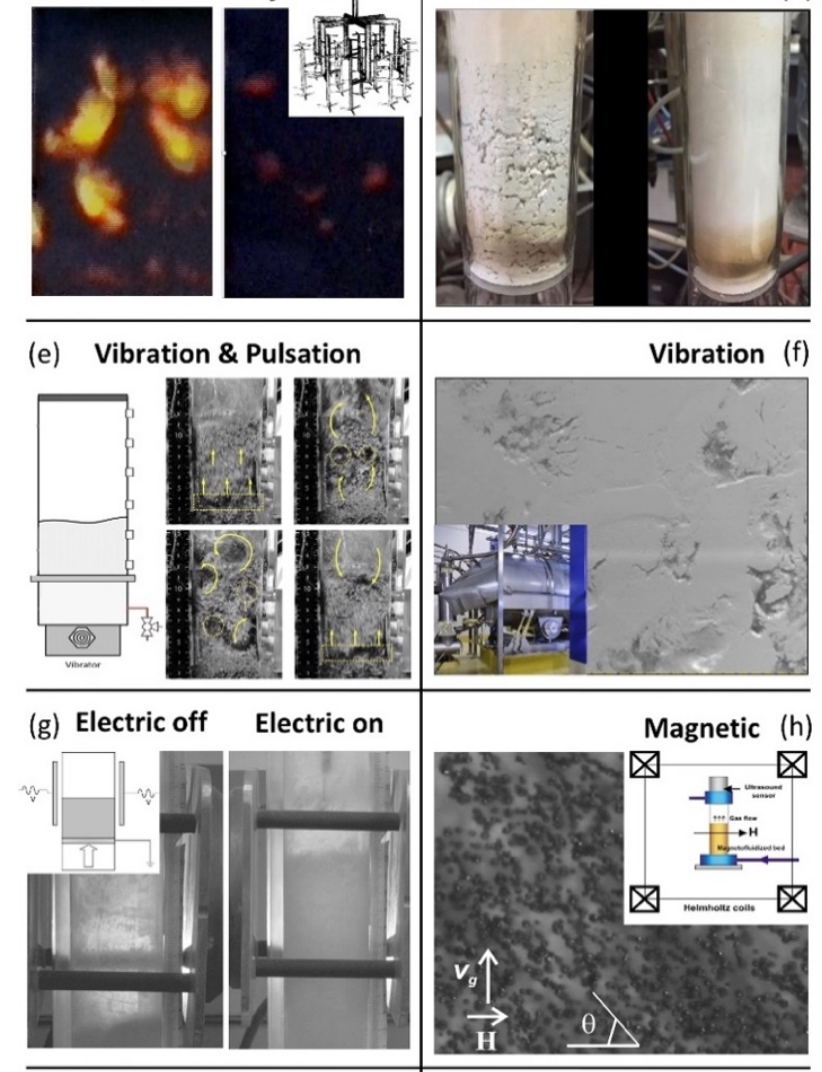

Magnetic (h)

(i) Vortex Flow
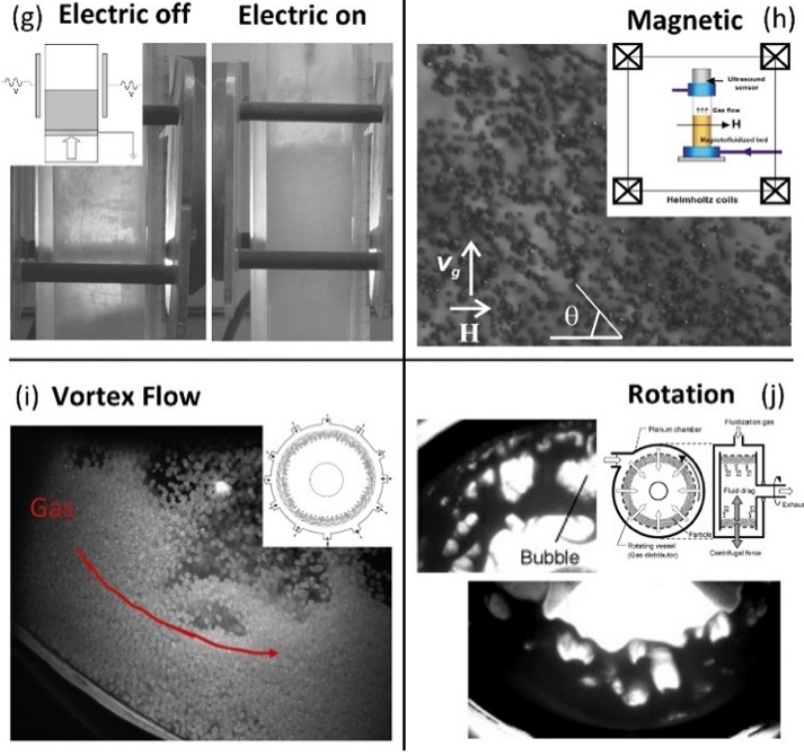

Figure 3: Examples of structured devices: (a) structured bubbling under pulsation, (b) study of incipient fluidization around internals [90], homogenization by applying (c) a fractal gas injector [76], (d) use of sound fluidizing fine natural limestone [60], (e) pulsation in a vibrating bed [69], and (f) an industrial vibrating fluidized bed dryer for milk powder (top view) [91], (g) electrofluidized bed of nanoparticles [65], (h) a magnetically stabilized fluidized bed [64], (i) a vortex chamber [82], and (j) a rotating fluidized bed [67]. Figures reproduced with permission, modified from the given references. 
more rigid mesostructure is detrimental to particle transport and, unlike traditional beds, characterized by vigorous mixing and isothermal operation, magnetically stabilized fluidized beds tend to present lower heat transfer coefficients [102]. Electrical stabilization induces a similar behavior. For example, semi-insulating particles become attractively or repulsively charged when polarized in the presence of an external electric field. The increase of the interparticle forces can then be used to control the formation of mesoscale structures and reduce bubble size under an AC or a DC field [103]. For example, Kleijn van Willigen et al. $[66,104]$ manipulated the flow in 2D and 3D beds of a Geldart A powder. They staggered grounded and live electrode wires shaped in an array with a volume density of $0.004 \%$, Figure $2 \mathrm{c}$, and reported a reduction in average bubble diameter under an $\mathrm{AC}$ field of up to $25 \%$, with a pronounced effect in the range of 5 - $20 \mathrm{~Hz}$, and up to $85 \%$ for Geldart B particles. Recent works point to the importance of electrostatic effects [105] in electrically stabilised small beds, long runs, and mixtures of materials prone to tribocharging. The application of an external force, however, is only possible in specific cases where the solid phase is "active" or a filler can be added to activate it, such as semi-insulating or ferromagnetic particles. In practice, structuring a full-size industrial bed with an external force field requires substantial additional energy, e.g., $100 \mathrm{~kW} / \mathrm{m}^{3}$ for magnetic $[40,66]$ or $40-80 \mathrm{~W} / \mathrm{m}^{3}[40$, 66] for electric actuation, respectively. The most viable technologies, for now, seem associated to a small particle scale, in particular nanofluidization [106], where an external field of low intensity can easily control the macroscopic flow $[65,107]$. For the most part, attempts to use external forces rely on setting a geometrical constraint, i.e., the spatial distribution of a body force field, but their dynamic component could be explored in more depth. There are promising studies on direct electrodynamic fluidization [108], scheduling magnetic actuation, such as rotational fields [109], and the use of transverse and longitudinal AC drives to control the mobility of granular media, demonstrating the ability to actuate a dynamic transition from a fluidized state to a creeping clogged state and a frozen clogged state [110].

In fact, some of the most successful alternatives to structure fluidized beds rely on dynamic, rather than geometrical constraints. There have been attempts to a direct, closed-loop control strategy, based on the manipulation of the gas flow to control the bubbling dynamics $[55,56,111,112]$, but given the system complexity, such closed-loop control is difficult to achieve at present. The use of open-loop control, via periodic excitation, is an alternative. Sound creates a dynamic pressure force that is strong enough to affect the fluidization behavior of a range of powders. High-intensity, low-frequency waves improve the fluidization of cohesive fine powders [113, 114], typically Geldart C and A, improving contact in carbon capture $[115,116]$ and utilization processes [60]. Sound waves also prominently feature in assisting the design of nanofluidized beds by controlling the expansion of the bed and the agglomerate size [59, 117]. However, they are usually insufficiently energetic to affect fluidization of coarser particles. This requires either 
mechanical energy or a particle-fluid force in the form of drag. In practice, mechanical vibration [118] and oscillating flows [119] are the two most popular non-intrusive ways to improve the fluidization of cohesive powders, by introducing additional energy simply in the form of alternating gas phase pressure and kinetic energy. Section 2 briefly summarizes the state-of-the-art on the use of pulsation and mechanical vibration in gas-solid fluidization. This is continued by a discussion on how oscillations initiate spatial patterns in shallow granular layers (Section 3), and how these can be propagated into a macroscopic structure (Section 4). Section 5 summarizes the theoretical and practical challenges associated with the creation and scale-up of a dynamically structured bed, and the conclusions in Section 6 provide a perspective on areas in which future work is encouraged.

\section{Pulsed and vibro-fluidization.}

Vibration is commonly used to improve fluidization behavior, particularly for cohesive powders. Horizontal, twist or vertical vibrations can be applied [120]. In a vertically vibrated bed, oscillating the bottom distributor or the entire vessel transmits mechanical energy into the bottom of the bed, from which it propagates into the bulk. How exactly it alters the gas-solid flow behavior has been the subject of debate. A packed bed below or at minimum fluidization transmits mechanical oscillations through bubbling [121], but, in a fluidized bed, the introduced energy dissipates quickly, due to inelastic collisions, and it has been argued that vibrations would not directly affect the upper part of the bed. However, vibration also introduces pressure and velocity fluctuations that can be transmitted through the bed, depending on the relative time scales of the perturbation and the particles' response. Detailed experimental and computational studies demonstrate that the magnitude of the acceleration is key to increase solid circulation rates above minimum fluidization conditions, which, in turn, affects the bubbling dynamics [122]. Generally, vibrating fluidized beds with Geldart B particles leads to a reduced bubble size [123], and homogenization [124]. Vibration also facilitates gas-solid mixing for Geldart D particles [70] and enhances segregation at low fluidization velocities [125]. Furthermore, it is used to improve handling fine powders, from differently sized nanoparticles [126] to Geldart A and C groups, where it is known to decrease the minimum fluidization velocity or the complete fluidization velocity [91]. Marco et al. demonstrated some of these benefits in the context of the oxidative coupling of methane, using vibration to reduce the agglomeration of $\mathrm{Li} / \mathrm{MgO}$ catalyst, which would have otherwise caused defluidization [127]. Beyond well-established drying applications, vibration allows fluidizing powders at lower pressures, which makes it a useful addition to facilitate coating, atomiclayer deposition or drying thermally sensitive products.

Instead of through mechanical action, a dynamic source of energy can be supplied through a fluid-particle force. Vibration requires the introduced energy to propagate through the bed, but an oscillating gas flow changes the fluid- 
particle force simultaneously throughout the entire volume, at least when operating at low Mach numbers (Figure 2b). Pulsation, which refers to the oscillation of the superficial inlet gas velocity, has been used to improve the performance of bubbling beds for years. This article is devoted specifically to avenues to use an oscillating gas flow to create dynamically structured systems, where the flow is fully predictable and can be manipulated externally to obtain different levels of mixing. However, pulsation and vibration have also been used extensively as ways to homogenize fluidization and improve powder mixing. For a more comprehensive list of applications involving the use of gas pulsation in fluidization technology, the reader is referred to recent reviews focused on this topic $[119,128]$.

The earliest documented research dates back to the 1960s, by Massimilla et al. [113], who used an on-off solenoid valve to create a pulsating airflow at frequencies from $1-10 \mathrm{~Hz}$. They distinguished three different fluidization regimes associated with the pulsation frequency: intermittent for $1.2-2.7 \mathrm{~Hz}$, piston-like for $2.7-4.8 \mathrm{~Hz}$ and plain fluidization for $4.8 \mathrm{~Hz}$ and above. Kobayashi et al. [129] controlled the gas flow to a cylindrical bed with the on-off ratio of a valve. Under pulsation, they observed considerably increased expansion and a higher pressure drop. In the 1970s, Wong and Baird [130] investigated the pressure fluctuations when using a pulsating flow with a frequency between 1 to $10 \mathrm{~Hz}$. In comparison with a constant flow, the gas retention time increased up to $51 \%$ when the air oscillated at the natural frequency of the bed. Following on from these early works, an increasing number of researchers have explored the effects of pulsation on performance, showing that it mitigates channelling in a broad range of fine powders [131] and reduces bubble size [132] as well as rising velocity [133]. For example, when pulsating Geldart A/B alumina particles in $15 \mathrm{~cm}$ deep cylindrical beds, Bizhaem and Tabrizi [134] observed a $25 \%$ reduction in the bubble size when increasing frequency from 1 to $4 \mathrm{~Hz}$. At higher values, i.e., $10 \mathrm{~Hz}$, the size becomes comparable to that under a constant flow, and is predicted using Werther's formulation [135]. The Mori-Wen correlation [136] has also been employed to estimate the bubble size in pulsed dryers [137] and heaters [138]. The rising velocity is predicted with reasonable accuracy using standard models. Li et al. [133] showed that bubble size and velocity are generally smaller for Geldart A than for Geldart B particles; they used the Shen et al. [139] and Davidson models [140] to predict the bubble velocity within $20 \%$ deviation from the experimental data.

The effects of pulsation are also affected by the polydispersity of the mixture. On the one hand, polydispersity can affect the nucleation process. Dong et al. [141] observed a reduction in the bubble size during the pulsation of a binary mixture of coal, due to the formation of groups of small bubbles close to each other rather than the independent larger elements observed under a constant gas flow. On the other hand, pulsation and vibration are themselves used to classify powders. For example, Saidi et al. [142] reported a $60 \%$ higher segregation efficiency of Geldart B mixtures applying on-off 
airflows to $6 \mathrm{~cm}$ deep beds of different binary mixtures, with better efficiency for increasing frequencies. However, the relation between segregation and frequency is complex [143] and other works report the opposite behavior [144]. Dong et al [145] reported an $80 \%$ improvement in the seggregation efficiency of fine coal particles when applying pulsation on a mechanically vibrated bed. A later computational work using a coarse-grained model [146] demonstrated that the gas pulsation suppresses the circulation of the heavy particles driven by bubbles and, as a result, it maximizes the separation.

Pulsation of the gas flow is typically achieved through the use of inlet valves before the plenum chamber, but other approaches are possible. Köksal and Vural introduced a moving double-plate distributor with two stacked reciprocating distributor plates, to create an oscillation in the inlet gas flow rate. They reported an approximately $40 \%$ drop in the bubble size for frequencies of $5-7 \mathrm{~Hz}$ with turnip seeds of $1.88 \mathrm{~mm}$ in average diameter, due to the frequent breakup of bubbles. In addition, they reported an enhancement in gas-solid contact, more uniform flow and lower expansions in quasi-2D cells filled with Geldart D turnip seeds and sand particles [147]. The different solid dynamics observed under a pulsating flow have a direct impact on the transport of heat and mass in a bed. As early as the 1960s, Bokun and Zabrodskii demonstrated that pulsed beds yield a similar heat transfer at 30-40\% lower volumetric flow rate than conventional beds [148]. More recent works quantify the improvement in both the heat transfer to the internals [149], and to the bed of solids [150], particularly below the minimum fluidization velocity, where heat transfer rates increase by a factor of 2 to 3 . Recent CFD-DEM investigations have looked in more detail into the effects of pulsation on the solid temperature distribution [151]. In drying applications, the use of gas pulsation has been established for a long time [152]. It breaks up interparticle forces, mitigates the formation of agglomerates and reduces the local mass transfer resistance. Akhavan et al. [153] showed how this leads to a significant increase in drying rates and a uniform mixing of lactose and cellulose fillers. They attributed the improvement in mass and heat transfer to a more homogeneous moisture distribution. Mixing time was reduced from $70 \mathrm{~min}$ in conventional operation to a few seconds under pulsation, and the required drying time was reduced by $20 \%$.

Recently, the Oak Ridge National Laboratory and the Universities of British Columbia and Tokyo have put forward a sustained systematic effort to bring pulsation into biomass energy production. Jia et al. have studied the benefits of drying biomass under pulsed flows with [69] and without additional vibration. Following in the steps of Wong and Baird [130], the authors associate pulsation at the natural frequency of the bed with the most efficient way to dry and mix Geldart D biomass particles, showing an improvement in the inter-phase mass [50, 137] and heat transfer [154] coefficients linked to a reduction in the apparent cohesiveness of the feedstock. 
Both pulsation and vibration facilitate many aspects of the operation of dryers and reactors: better homogenization, mixing, processability, and gas-solid contact. However, it is important to note that most of these effects are correlated to specific particles, operation conditions, and the unit scale and geometry. They lack universality and, for that reason, can be hard to reproduce. In order to formulate new robust designs, it would be helpful to create a scalable and more responsive flow structure that one could manipulate externally. Steps to realize this by using pulsation to dynamically structure fluidized beds and thus intensify their operation, is what the next sections focus on.

\section{Initiation of dynamic patterns in granular layers.}

Understanding how surface patterns emerge is the first step to expand them into a structured flow to control mixing in dryers, coaters or reactors. Nature provides many examples of the response of granular matter to a dynamic oscillation. Surface features in deserts and sediment beds respond to the dissipation of energy under the action of a dynamic force, in this case, a fluid force inferred on the particles that contains a full spectrum of temporal and spatial scales, such as turbulent boundary layers in the wind, riverbeds or coastal tides (Figure 4a). These phenomena are studied throughout different areas of physics, from aeolian transport in planetary sciences [155] to snow [156] or sediment dynamics [157], but, at a fundamental level, why and how a granular system self-assembles into a spatial pattern has attracted particular interest [158]. It is well known that, under a periodic oscillation, granular layers organise into surface waves [159]. From a phenomenological point of view, this response has been described in terms of combinations of harmonic oscillators subject to energy input and damping forces (Figure 4b) [160], but the pattern as a whole is really a reflection of collective, emergent systems behavior. At a small scale, the patterns can be easily reproduced in experiments, yet, they pose a significant challenge to theoreticians. For that reason, they have been adopted as a convenient benchmark to test new physical models.

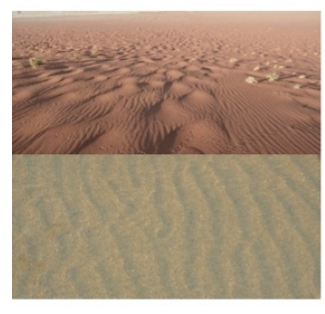

(a)

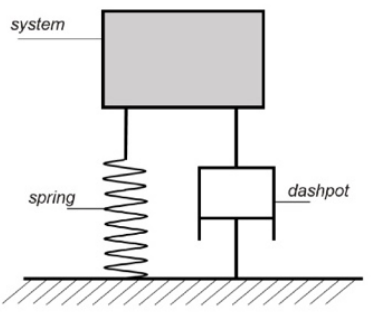

(b)

Figure 4: (a) Examples of spatial patterns arising in natural granular systems. Photographs by MO Coppens, taken in the Namib desert and on Uvita Beach, Costa Rica. (b) Phenomenological description: mechanical oscillator. 


\subsection{Vibration-driven surface patterns}

Vibrated shallow granular layers have been studied for decades. In the late 1980s, Douady et al. observed how a subharmonic wave appears in 2D geometries when a granular layer is vertically shaken in ambient air [161]. Soon after this, Melo et al. showed the remarkable variety of possible patterns in 3D (Figure 5a) [162]. The authors attributed the formation of a regular pattern to an intrinsic mode of the oscillatory system, due to the collective manifestation of the energy dissipated in the impacts [163].

This type of macroscopic surface features are reminiscent of the standing waves, created in vertically vibrated fluids, termed Faraday instability [164]. Faraday waves correspond to a parametric resonance phenomenon, which arises naturally on fluid surfaces from the governing principles of viscous fluids [165]. However, in a granular system, nonlinear dissipation of energy, interparticle friction and the absence of surface tension mark qualitative differences that enrich the range of possible configurations.

Working with a powder, distinctive transitions between different types of patterns are observed (Figures 5 and 6). Reproducing each regime is often used as a qualitative test for new theoretical frameworks, while the prediction of the wavelength and the pattern morphology provide a more rigorous quantification $[162,163,166]$. The scale of the pattern or wavelength is associated with the lateral motion of particles and the moving time, which results from a balance between the input energy and the dissipation through collisions and friction $[167,168]$. Under vibration, the wavelength of the pattern decreases with increasing frequency, until it saturates at large values, which depends on particle characteristics and layer thickness [169-171]. Saturation has been associated with the velocity at which the energy input propagates through the layer, and, therefore, the saturation frequency varies with the layer thickness, but not so much with the particle diameter [172]. Despite differences between fluids and granular matter, Umbanhowar et al. reported a linear relationship between the wavelength and the inverse of vibration frequency squared, similar to the Faraday instability in vibrating liquids [166]. The onset of pattern formation in granular systems has been studied with dimensionless analysis using a Froude number, $\Gamma=\Gamma_{v}$, to quantify the ratio of the maximum driving and damping accelerations under vibration:

$$
\Gamma_{v}=\frac{4 \pi^{2} f^{2} B}{g}
$$

where $f$ is the vibration frequency, $B$ is the amplitude, and $g$ is the gravitational acceleration. A thin granular layer would be on the verge of detaching from the vibrating plate at $\Gamma_{v}=1$, where the maximum acceleration caused by vibration is 
a)
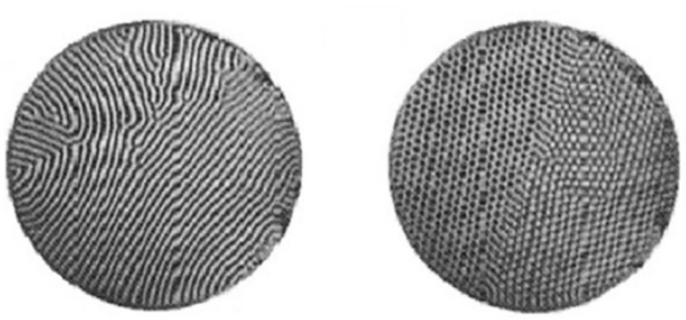

b)
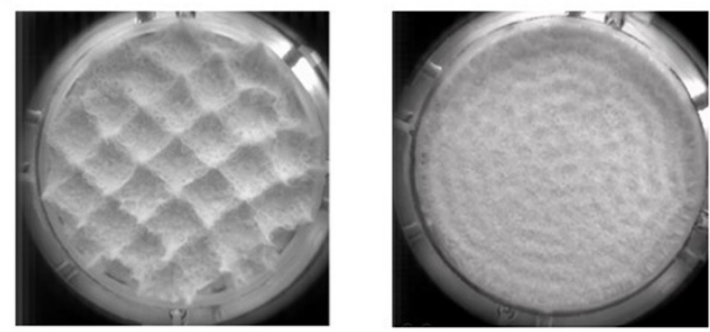

Figure 5: Examples of dynamic patterns in shallow granular layers. (a) Squares and stripes in a vibrating layer in the presence of air; reproduced with permission from [163]. (b) Squares and concentric circles in granular layers under an oscillating gas flow.

balanced by gravity. The type of patterns displayed, e.g., squares, stripes, hexagons, spirals and local "oscillons" (soliton waves), depends on the frequency and the Froude number $[170,173,174]$. A periodic structure appears beyond a given onset, termed as critical $\Gamma_{v, c}$, which is a strong function of the presence of air. When reaching $\Gamma_{v, c}$, a vibrated granular layer displays a square grid across the bottom of the container, maintaining an identical wavelength in both orthogonal directions. As $\Gamma_{v}$ continues to increase, stripes with a smaller wavelength gradually replace the squares, and a strong curvature starts to appear near the walls [166]. Umbanhowar and Swinney [172] found that the frequency for a squarestripe transition is inversely proportional to the bed height and the particle diameter squared. At a higher acceleration, $\sim \Gamma_{v}>4$, both squares and stripes are unstable, and the pattern transforms into a grid of hexagons (Figure 6a). The set of isolated peaks on a triangular lattice moves towards the centres of hexagonal cells on the next oscillation, and vice versa. Melo et al. also showed that hexagonal patterns repeat at a time scale that is not strictly subharmonic [163]. They studied a granular system purely in terms of the response of a layer of hard particles to an external acceleration. The way the bed transmits and dissipates energy, however, also depends on interparticle contacts and their interaction forces. Since $\Gamma_{v}$ does not take any particle property into account, it is not surprising that the same $\Gamma_{v, \mathrm{c}}$ cannot describe different powders. Goldman et al. studied the relationship between the onset of pattern formation and particle properties [175]. They reduced interparticle friction by introducing fine smooth graphite particles and found that lower levels of friction destabilize the patterns to the point of collapse. By studying the dilation of the granular layer surface and the intensity of the solid-plate collisions, Mujica and Melo found that a change from solid-like to liquid-like behavior occurs prior to the formation of a pattern [176]. They pointed out that the dilation process is linked directly to the onset of the pattern formation, as it allows the kinetic fluctuations of particles to be initiated and develop. 
Numerical studies provide further insight into some of these phenomena, such as the role of individual particle motion, interparticle contact forces and collision time. Using discrete models, Aoki et al. found that the presence of interparticle friction is necessary to reproduce numerically the formation of convection rolls in a 2D domain [177]. This model was progressed by Bizon et al., who assigned a velocity-dependent impact restitution coefficient [178]. Luding et al. used a 2D hard-sphere discrete element method (DEM) [169] to capture the formation of alternating waves, i.e., $\Gamma_{v}=2.6-4.3$, $f=8-14 \mathrm{~Hz}$. They noted that the waves were only stable in the presence of grain-plate friction and would otherwise collapse. This was attributed to increased lateral mobility of the peaks in the granular wave that destabilizes the structure. Bizon et al. extended the work to 3D [179] with quantitative agreement for all the phase regimes proposed by Melo et al. [163] (Figure 6).

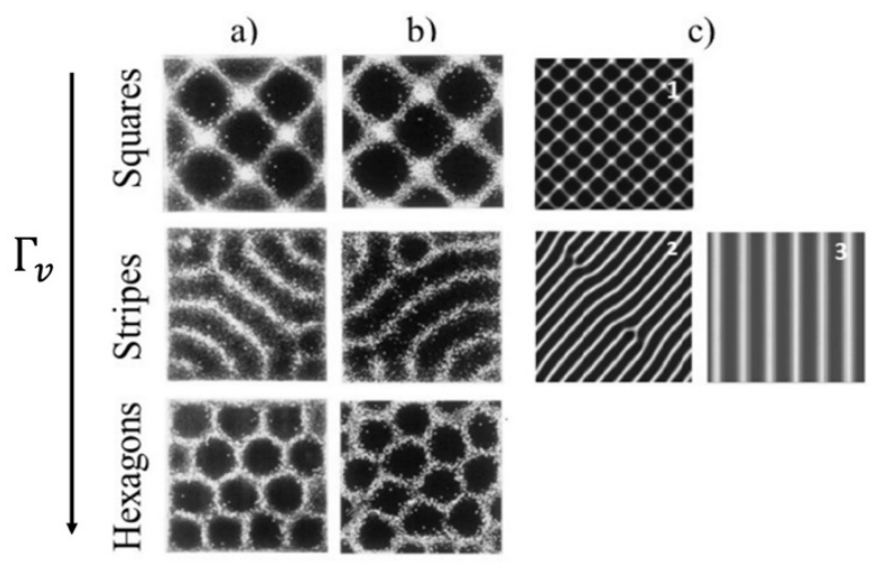

Figure 6: Pattern regimes in vibrated shallow granular layers. Brightness indicates the height of the granular layer. (a) Experimental observations, using lead spheres with diameter 0.5-0.6 mm. Numerically obtained regimes, using (b) discrete and (c) continuum models. Reproduced with permission from [179-181].

Hydrodynamic models have also been used to study granular patterns, treating the solid phase as a continuum. In a purely granular flow (i.e., neglecting surrounding air) a first coarse-grained hydrodynamic description can be obtained by modifying the Boltzmann-Enskog equation with the appropriate Chapman-Enskog procedure [182, 183]. The hydrodynamic equations then comprise continuity, momentum and energy conservation equations, but the system needs to be closed constitutively. Cerda et al. [184] simplified the continuity and momentum conservation equations by assuming that particles attain a horizontal velocity, $v_{\perp}$, proportional to the local gradient of the layer thickness, $h(x, 0)$, i.e., $v_{\perp}(x, 0)=v_{0} \nabla_{\perp} h(x, 0)$, where $v_{0}$ is the collision velocity during grain-plate impact. They demonstrated that in a pattern of squares (Figure 6c-1) the layer dilates and redistributes the mass during the period of free flight, acting like a typical diffusion process. Park and Moon [181] followed up on this model with an effective solid pressure, $p$, which is 
a function of the solid velocity field, i.e., $p=\alpha \rho|\nabla \cdot v| \nabla \cdot v$, where $\alpha$ is a system coefficient, as a way to implement a saturation mechanism for the lateral motion that results in the instability responsible for a pattern of stripes (Figure 6c2). This alteration allowed them to reproduce the transition from stable square to stripe patterns.

Building on these works, Bougie et al. [180] were able to reproduce these patterns by incorporating the transport of granular energy from the kinetic theory of granular flow (KTGF) in a 2D domain. They observed that, during the vibration, a shock wave arises from the contact between falling particles and the plate. This wave propagates supersonically through the layers in all directions and initializes the lateral movement of the grains. Bougie and Duckert extended their study from $2 \mathrm{D}$ at $\Gamma=2[180]$ to a $3 \mathrm{D}$ system and a wider range of $\Gamma$ [185]. They replicated the pattern wavelength in a striped pattern, i.e., the separation between the stripes in Figure 6a and 6c-3, but they were not able to replicate the square and the hexagon typologies for $\Gamma_{v}>4$. In contrast, discrete models did capture the full variety of patterns, which, alongside the work of other authors $[169,177]$, suggests that the interparticle friction plays a role in the generation of some typologies.

\subsection{Gas-driven surface patterns}

Pulsation introduces energy via the fluid-solid interaction force using a pulsed superficial gas velocity, $u$, which oscillates according to:

$$
u=u_{\min }+u_{a}[1+\sin (2 \pi f t)]
$$

where $f$ is the frequency of the oscillation applied, $u_{\text {min }}$ is the minimum gas superficial velocity and $u_{a}$ is the amplitude of the pulse. The resulting time averaged velocity is $\bar{u}=u_{\min }+u_{a}$. Alternatively, this equation can be rewritten in terms of dimensionless velocities, after division by the minimum fluidization velocity $u_{\mathrm{mf}}$ of the powder as $\hat{u}=u / u_{\mathrm{mf}}$ :

$$
\hat{u}=\hat{u}_{\min }+\hat{u}_{a}[1+\sin (2 \pi f t)]
$$

In a pulsed system, the gas fills interparticle voids and imposes drag directly and simultaneously onto all particles in the volume. Since it entails more complex physics, gas-driven pattern formation (Figure 5b) is not as well understood. Coppens et al. first demonstrated that, using an oscillating gas flow through a wide and shallow granular layer, patterns of a very similar nature to vertically vibrating layers are observed [186]. Li et al. then studied the phase diagrams, characterized by the formation of squares and stripes at the lower and higher end of the frequency range [187]. The authors claimed that a large pressure fluctuation appears in the frequency range associated with the formation of a 
pattern. As in mechanically vibrated systems, the wavelength of subharmonic squares and stripes decreases with increasing pulsating frequency. In fact, these features also follow behavior consistent with a Faraday instability, whereby the wavelength of the spatial pattern is inversely proportional to the square of the perturbation frequency (Figure 7). More recently, de Martín et al. [188] investigated the onset of pattern formation across different powders recognizing that the response time of a collection of particles to a drag force must be linked to their size.

They attributed the pattern formation to a secondary instability resulting from the propagation of voidage waves induced by the gas pulsation. de Martín et al. proposed a dimensionless hydrodynamic number to describe the onset for gasdriven pattern formation in shallow layers of Geldart B particles [71].

$$
\Gamma_{g s}=\frac{u_{a}}{u_{t} \overline{\phi_{s}}}
$$

where $u_{t}$ is the terminal velocity of particles (used as a proxy for the particle response time), and $\bar{\phi}$ is the time-averaged solid fraction. A pattern arises beyond a critical amplitude and collapses below it, but, unlike vibrated layers, the critical amplitude depends strongly on the pulse frequency. This is addressed by the use of the non-dimensional time scale whereby the pulse frequency is normalized with the system's natural frequency $f_{n}$ given by Baskakov et al. [189] to define another parameter that takes into account the bulk dynamics of the layer. Applying these dimensionless numbers, a universal instability curve can be drawn, describing the onset of pattern formation and separating two regimes, corresponding to stable and unstable patterns (Figure 8). The critical amplitude decreases dramatically when approaching $f / f_{\mathrm{n}} \sim 0.5$, reaching a plateau for $f / f_{\mathrm{n}}=1$, which suggests a parametric resonance between the formation of kinematic waves and the bulk dynamics [188].

The numerical reproduction of gas-driven patterns is very complex, because it relies on capturing a subtle interfacial coupling. Successful simulations have been reported in relatively deep quasi-2D systems [51], but, in shallow layers, one faces a tougher challenge in defining the simulation grid and selecting the drag model, to ensure a good prediction of the minimum fluidization velocity. The grid used for the fluid must be sufficiently fine to describe the momentum exchange, but also ensure a good averaging of the solid phase properties. As in vibrating systems, a continuum model struggles to reproduce formation of several pattern types. Nonetheless, the stability curve could be reproduced qualitatively using a 1D particle array model [190], which simulates the dynamics of a string of vertically stacked particles [191] and, quantitatively, using 2D discrete particle simulations (Figures 8 and 9). 


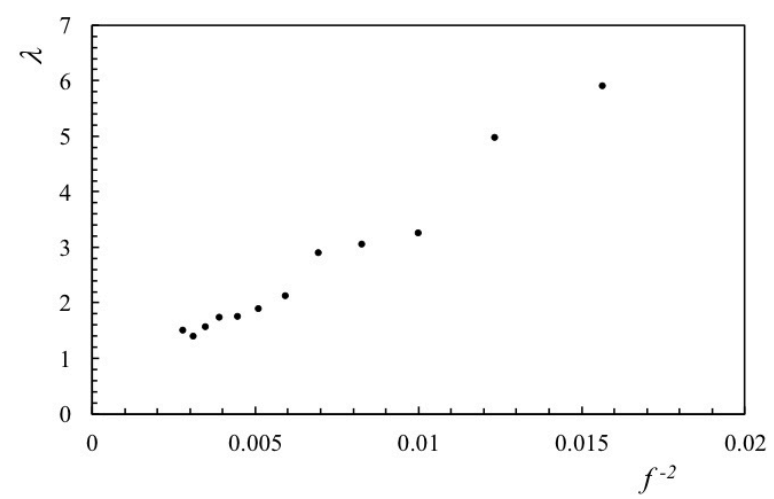

Figure 7: Variation of the wavelength of surface features in shallow 3D granular layers of 238 um glass beads under a pulsed gas flow. Experiments were carried out in a cylindrical bed of $14 \mathrm{~cm}$ diameter, height $h=3 \mathrm{~mm}\left(12 \mathrm{D}_{\mathrm{p}}\right)$, dimensionless gas superficial velocity $\hat{u}=u / u_{\mathrm{mf}}=0.6+0.8[1+\sin (2 \pi f t)]$.

Discrete simulations were conducted using open source software, CFDEM [192] and spherical glass beads of different sizes. Mechanical properties and numerical settings followed those validated in 3D simulations of a pulsating bed in [51]. Continuum simulations were also conducted using a classic hydrodynamic model based on the KTGF and a frictional stress model in the tw o-fluid model framework, TFM. Both computational frameworks reproduce the formed pattern and its general attributes, such as the inverse correlation between the pattern wavelength and the pulse frequency

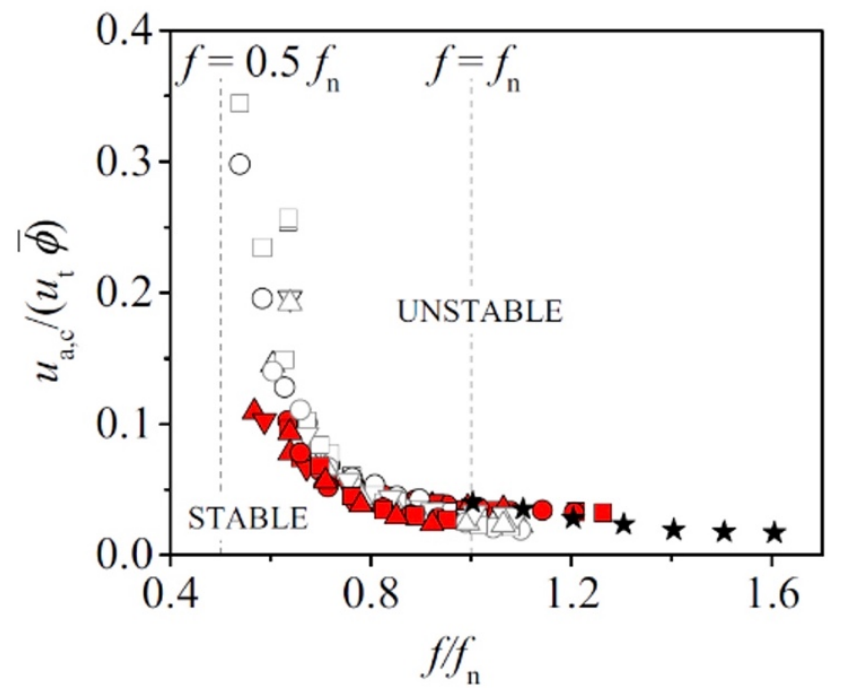

Figure 8: Universal stability curve for pattern formation in shallow, 3D pulsed fluidized beds, of Geldart B particles under a pulsed flow with gas superficial velocity $u=u_{\min }+u_{a}[1+\sin (2 \pi f t)]$, where the amplitude $u_{a}$ that leads to the onset of pattern formation is denoted $u_{a, c}$. Particles include spherical glass beads of $130 \mu$ m diameter with $u_{\min }=3$ or $4 \mathrm{~cm} / \mathrm{s}\left(\hat{u}_{\min }=u_{\min } / u_{m f}=\right.$ 2.5 or 3.3) and height $h=2-7 \mathrm{~mm}$ (white symbols), glass beads of $240 \mu \mathrm{m}$ diameter with $u_{\min }=3 \mathrm{or} 6 \mathrm{~cm} / \mathrm{s}\left(\hat{u}_{\min }=0.75\right.$ or 1.5$)$ and $h=3-7 \mathrm{~mm}$ (red symbols), and polystyrene particles with a diameter of $600 \mu \mathrm{m}$ at $u_{\min }=11.5 \mathrm{~cm} / \mathrm{s}$ and $\mathrm{h}=10 \mathrm{~mm}$ (stars). Reproduced with permission from [188]. 


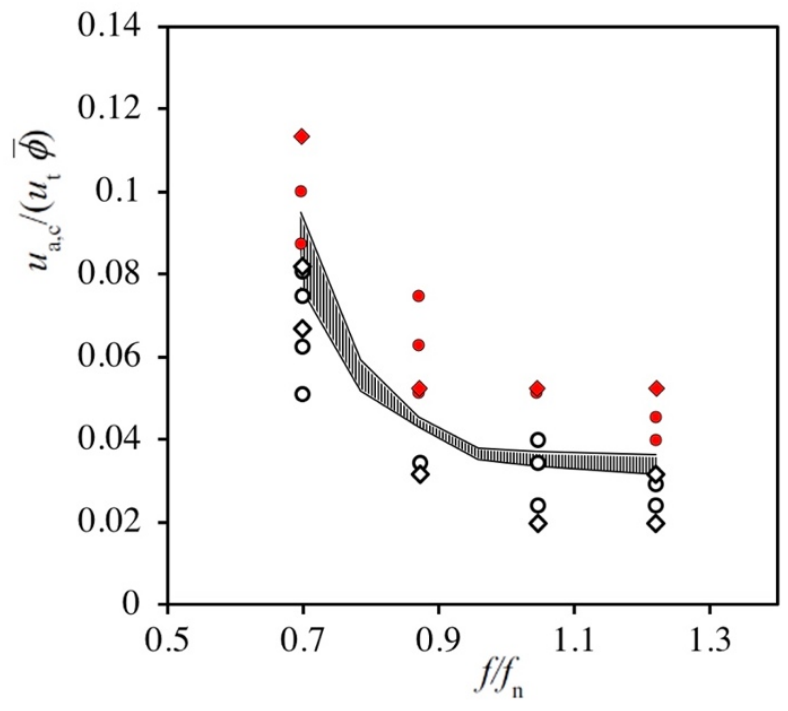

Figure 9: CFD-DEM reproduction of the onset for pattern formation reported in de Martin et al. [188]. Grey area denotes the experimental threshold for the critical amplitude $u_{a, c}$ for several replicates of $128 \mu \mathrm{m}$ and $238 \mu \mathrm{m}$ glass beads in a bed of height $h$ $=7 \mathrm{~mm}\left(29 D_{p}\right)$ and width $15 \mathrm{~cm}\left(630 D_{p}\right)$. The gas flow is pulsed with superficial velocity $u=u_{\min }+u_{a}[1+\sin (2 \pi f t)]$, with $u_{\text {min }}$ $=4 \mathrm{~cm} / \mathrm{s}$. Simulation set-up as described by Wu et al. [51]. Circle and diamond symbols correspond to 2D simulations of 128 um and $238 \mu \mathrm{m}$ particles, respectively. Closed symbols (• and $\bullet$ ) show the computations that predict pattern formation, and open symbols $(\circ$ and $\diamond)$ those that do not.

The same phenomenon is observed in 2D and 3D domains using different open source codes, such as MFIX [193] and CFDEM [192]. Figure 10 depicts examples of the surface wave in a shallow quasi-2D bed, and Figure 11 shows the more complex surface configurations observed in 3D shallow layers. Fluidized at the same conditions, the computational system develops a comparable pattern and wavelength in both 2D and 3D systems. Discrete simulations of $238 \mu \mathrm{m}$ monodispersed spherical particles are able to capture variations in the pattern (Figure 11). Nevertheless, the variety of surface features has not yet been reproduced with a classic implementation of the TFM under the same conditions of pulsed shallow layers.

Although the patterns in pulsed gas-fluidized and vertically vibrated granular layers look alike, they are driven by different ways of gaining and dissipating energy. The stability condition in Figure 8 depends on $\Gamma_{\mathrm{c}}$ and $f / f_{\mathrm{n}}$; both are independent of the particle shape and the apparent solid friction. Experiments and 2D simulations display patterns that are independent of the frictional properties of the particles and both discrete and hydrodynamic models convincingly capture the formation of surface waves. However, the continuum formulation shows quantitative errors in $2 \mathrm{D}$ and still struggles to recreate how a pattern emerges in 3D. Studies of vibrated particle layers associated solid friction to the 
pattern type and variety $[167-169,185]$, suggesting that it is responsible for maintaining stable pattern wavelengths and creating different configurations.

\section{Propagation of dynamic patterns in a fluidized bed.}

Vibration and pulsation are used extensively in deep granular beds [194-196], but, on their own, they do not create a reproducible flow structure, because the surface patterns that emerge in a shallow layer quickly collapse in deeper layers. squared, as well as its independence from the domain width. The discrete model shows quantitative agreement with the experimentally observed wavelength, but the continuum model overpredicts it, see Figure 10. It is worth noticing that the stability curve shown in Figure 9 can be reproduced by assigning no interparticle friction, which is an indication that friction plays a minor role, or no role at all in the onset of pattern formation.

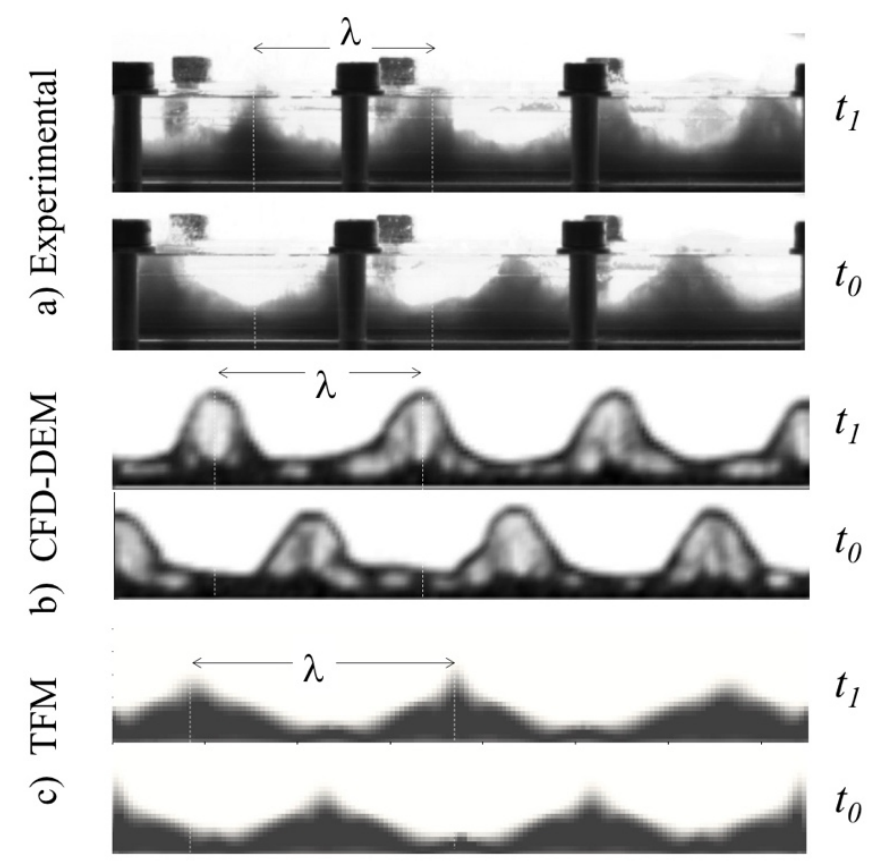

Figure 10: Gas-driven patterns in a shallow, $2 D$ domain. Particles are $238 \mu \mathrm{m}$ glass beads. Bed height $h$ is $7 \mathrm{~mm}\left(29 D_{p}\right)$. Dimensionless gas superficial velocity $\hat{u}=u / u_{\mathrm{mf}}=1.0+2.0[1+\sin (2 \pi 8 t)]$.(a) Experimental patterns in a bed of $45 \mathrm{~cm} \times$ $0.7 \mathrm{~cm} \times 1 \mathrm{~cm}$; (b) Results from CFD-DEM, using CFDEM; (c) TFM, using MFIX. Top and bottom show patterns formed during consecutive pulses, $t_{1}=t_{0}+1 / f$. 


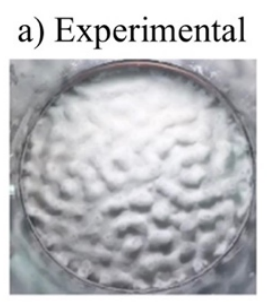

b) Numerical CFD/DEM
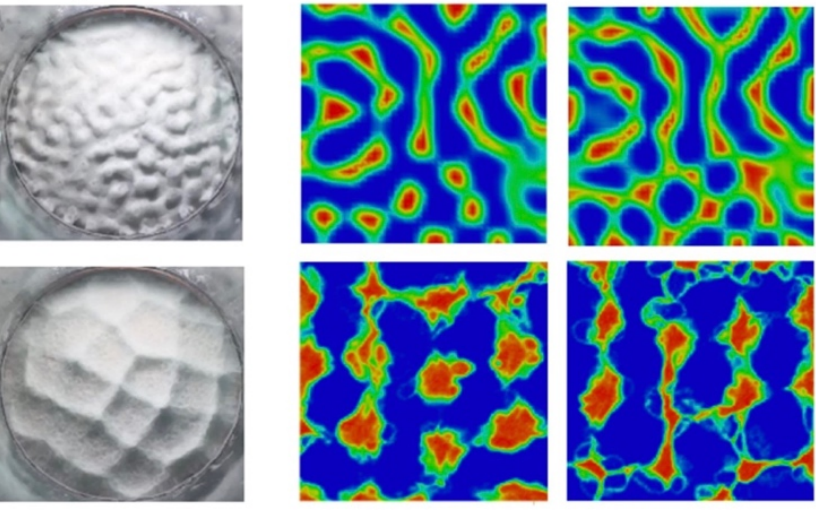

Figure 11: Gas-driven patterns in a 3D domain, with $238 \mu \mathrm{m}$ glass beads and a bed height $\mathrm{h}$ of $3 \mathrm{~mm}\left(29 \mathrm{D}_{p}\right)$. (a) Experiments in a cylindrical unit of $14 \mathrm{~cm}$ diameter (b) CFD-DEM in an $8 \times 8 \mathrm{~cm}$ rectangular unit. Dimensionless gas superficial velocity $\hat{u}=$ $u / u_{\mathrm{mf}}=0.6+0.8[1+\sin (2 \pi 20 t)]$ and $\hat{u}=u / u_{\mathrm{mf}}=0.6+1.4[1+\sin (2 \pi 12 t)]$ for the top and bottom patterns, respectively.

Umbanhowar et al. [174] reported surface patterns in vibrating layers in a range from 3 to 30 particle diameters for Geldart B particles, and Melo et al. documented patterns up to 40 particle diameters in Geldart A to D [163]. Beyond that, the surface pattern disappears, because the energy introduced at the bottom cannot propagate far and high enough without damping, due to inelastic collisions. For this reason, vibro-assisted beds are regarded as a way to enhance mixing, rather than a good method to impose a large-scale flow structure. Using the particle-fluid force as the source of energy instead, one is no longer restricted to a planar source. The dissipation of energy in collisions still defines the length scale in the response of single particles and the formation of coherent flow structures, but, because the entire system is excited simultaneously, the dynamics of the perturbation induce a collective, emergent response in the solid particles.

The surface waves presented earlier for pulsating layers survive up to a height of around 40 particle diameters for Geldart B particles. Increasing the height further introduces a new phenomenon characteristic of gas-driven fluidized systems, because the expansion and dilution of the layer lead to slugging or bubbling. Under a constant gas flow, the gas-solid emulsion becomes unstable - a primary hydrodynamic instability - leading to phase separation. Bubbles are nucleated at the bottom of the bed and their motion and subsequent interaction become poorly predictable. The study of the pressure fluctuations with nonlinear analysis methods, such as chaos analysis [12] and wavelet analysis, allows for a certain characterization of the response of the bed and reveals the characteristic frequencies associated to key events, e.g., bubbling, bubble rupture, coalescence, and breakage. For example, the time scale of fluctuations due to bubbling is a function of the particle size and shape, the bed geometry and the static bed height [197]. One can also try to 
characterize the intrinsic behavior of a bed by looking at it as a mechanical vibration oscillator (Figure 4b), deriving natural frequencies, $f_{n}$. Different formulations have been proposed, based on the source of the pressure fluctuation, e.g., generation of surface waves, bubble formation, coalescence and eruption, or oscillations in the plenum [197]. Their expressions rely on a force balance that considers either a system comprised of single particles or layers of particles with a given void fraction. Perhaps the most widely used is that of Baskakov et al. [189], who describe the system as an ideal liquid in a U-shaped tube of constant cross-sectional area, resulting in:

$$
f_{n}=\frac{1}{\pi} \sqrt{\frac{g}{h_{m f}}}
$$

Hao and Bi [160] define the natural frequency by treating a fluidized bed as a second-order, damped vibrating system, so that:

$$
f_{n}=\frac{\sqrt{1-\zeta^{2}}}{2 \pi} \sqrt{\frac{g}{h_{m f}}}
$$

where $g$ is the gravitational acceleration, $h_{m f}$ is the height of the bed at the minimum fluidized state, and $\zeta$ is the damping factor associated with the system. In both models, the predicted natural frequency decreases with increasing static bed height.

When the primary bubbling instability progresses in the presence of a pulsed gas flow, both interact and give rise to a very interesting transition. Figure 12 shows an example of how the surface wave in a shallow layer transitions to bubbling as the static height increases. The data are obtained in a quasi-2D cell made of Perspex $(45 \mathrm{~cm} \times 5 \mathrm{~cm} \times 1 \mathrm{~cm})$, described in previous works [51] and the gas superficial velocity oscillates according to Eqs (2) and (3).

Most of the time, the patterns that appear in a pulsed shallow layer collapse in a deeper bed, because the stresses created by the motion of the bubbles dominate the motion of the solids and supersede the surface wave created by the oscillations. However, under suitable conditions, both phenomena interact, and the solid circulation induced by the pulsation contains the primary hydrodynamic instability, preventing chaotic bubble motion. Bubbles are indeed created, but only at specific nodes and at specific times, self-organizing into a macroscopic pattern. Figure 12 shows this incipient phenomenon in a shallow bed, transitioning from a surface wave to a bubbling bed. Within a specific, continuous range of $\hat{u}_{\min }, \hat{u}_{\mathrm{a}}$ and $f$, the bubble nucleation becomes reproducible and the bubbling fluidized bed is said to be "dynamically structured". Gas bubbles are formed at specific nodes, spaced regularly by a characteristic transversal distance or wavelength $\lambda$, and a correlation appears between the nucleation sites in consecutive pulses, whereby the nodes in every 
pulse shift laterally by $\lambda / 2$. Furthermore, this arrangement can propagate through a deep bed, leading to a fully predictable flow structure, see Figure 3a, where bubbles do not move laterally, coalesce or break. It is important to note that this phenomenon is not the result of a linear combination and propagation of waves in the domain. The wavelength of the waves shown in Figure 12 and of the bubble patterns in Figure 13 are independent of the width of the domain, at
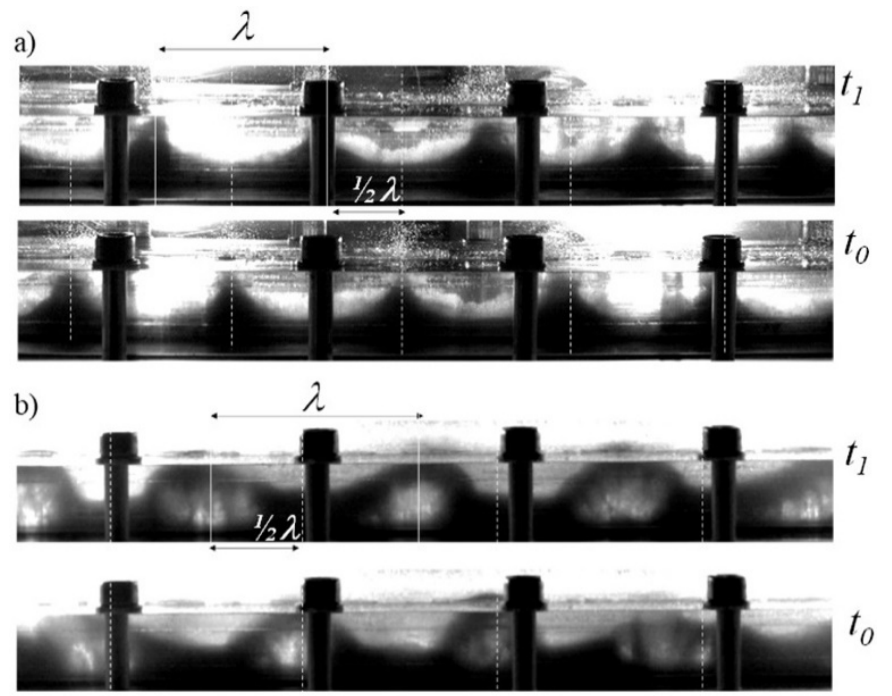

Figure 12: Transition from a surface wave to structured bubbling for increasing height, using $238 \mu$ m glass beads, with $u_{\mathrm{mf}}=4.1$ $\mathrm{cm} / \mathrm{s}$. Top and bottom frames in both a) and b) show patterns formed during consecutive pulses, to and $t_{1}=t_{0}+1 / f$. Dimensionless gas superficial velocity $\hat{u}=u / u_{\mathrm{mf}}=1.0+2.0[1+\sin (2 \pi 10 t)]$ for bed heights $h$ of $(a) 7 \mathrm{~mm}\left(29 D_{p}\right)$ and (b) $1 \mathrm{~cm}\left(42 D_{p}\right)$
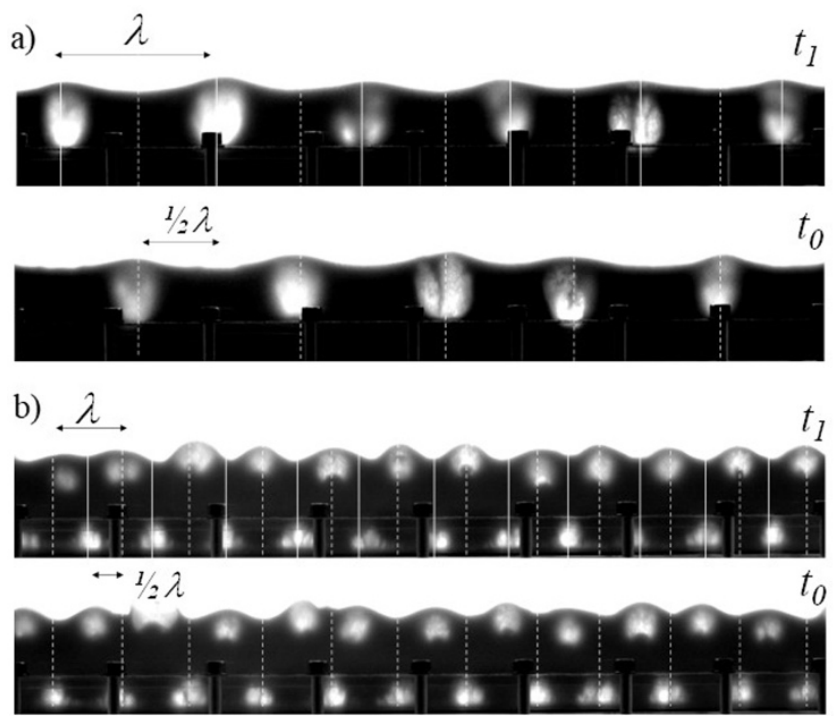

Figure 13: Structured flow of bubbles when pulsating the air flow. The frequency affects the bubble size $D_{b}$ and the wavelength $\lambda$.

The particles are $238 \mu \mathrm{m}$ glass beads, with $u_{\mathrm{mf}}=4.1 \mathrm{~cm} / \mathrm{s}$. The stills show bubble patterns show patterns formed during consecutive pulses, to and $t_{1}=t_{0}+1 / f$. Bed height $h$ of $4.5 \mathrm{~cm}\left(189 D_{p}\right)$ and non-dimensional gas superficial velocity $\hat{u}=u / u_{\mathrm{mf}}=0.5+$ $2.14[1+\sin (2 \pi f t)]$ for (a) $f=5 \mathrm{~Hz},(b) f=7 \mathrm{~Hz}$. 
least in a sufficiently wide bed [186]. Both the initiation and the propagation of this phenomenon lead to a sub-harmonic pattern, recurring at half of the perturbation frequency.

Figures 13, 14 and 16 show examples of the bubble dynamics for different pulsating frequencies $f$. In this case, the natural frequency, $f_{n}$, computed from Eq. (5), is $3.1 \mathrm{~Hz}$, and the pressure signal shows a maximum spectral density within a range of 2.5 to $6 \mathrm{~Hz}$. When operating at the lower end of this range of $f, 3-4 \mathrm{~Hz}$, bubbles stagger in separate rows in response to each pulse, but their spatial distribution remains unstructured in the horizontal direction: they move laterally, coalesce and break, rising at different velocities. When operating at higher frequencies $f$, say 9 or $10 \mathrm{~Hz}$ (Figure 14), much smaller bubbles emerge that are spatially randomly distributed, in a comparable way to a fluidized bed under constant gas flow. The use of pulsation at such frequencies could improve the performance of fluidized bed processes, because it reduces bubble size and enhances the powder flowability, by introducing a new source of stress in the solid phase. However, one loses control over the bubble size and, as a consequence, any ability to manipulate mixing and transport. Therefore, while operating in the unstructured regime might still offer benefits, it lacks general applicability and involves uncertainties in design and scale-up. The optimal conditions to pulse a bed in order to homogenize bubbling are system and scale-dependent, e.g., due to their association to the bed's natural frequency [137].

Earlier, in Eq. (4) and Figure 8, it was shown how patterns in a shallow layer emerge beyond a certain threshold, which is a function of the response time of the solids phase, and is characterized by the terminal velocity of the particles, $u_{\mathrm{t}}$ [188]. For a shallow layer of height, $h$, fluidized by a pulsating flow over a minimum flowrate, $u_{\min }$, and frequency, $f$, a regular surface wave emerges above a critical pulsation amplitude, $u_{\mathrm{a}, \mathrm{c}}$, and collapses only at large amplitudes. Bubble patterns in deeper layers behave differently: a stable pattern emerges only within a moderate range of $f$ and $u_{\mathrm{a}}$. Outside of this domain, bubbling progresses in a chaotic manner, similar to the operation at constant gas velocity. Within it, a reproducible pattern emerges, and key features, such as bubble size and separation become strongly correlated and are a function of the pulsation characteristics. Crucially, changes in frequency $f$ and amplitude $u_{\mathrm{a}}$ allow one to change the way the gas is allocated into bubbles, across a finer or coarser lattice, which affects both the pattern wavelength and bubble size.

In vibrating [166] and pulsed shallow layers, the wavelength $\lambda$ is inversely proportional to the square of the reciprocal of the frequency, $f^{-2}$ (Figure 7). A macroscopic bubble pattern shows the same qualitative trend, whereby an increase in $f$ reduces the separation between bubbles, $\lambda$ (Figure 13). However, the phenomenon is no longer directly comparable to 
a) Unstructured pulsation - Low $f$

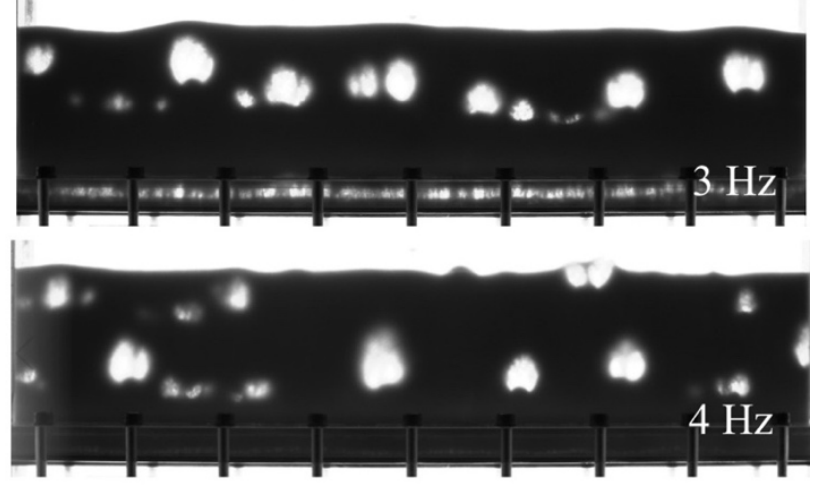

b) Unstructured pulsation - $\operatorname{High} f$

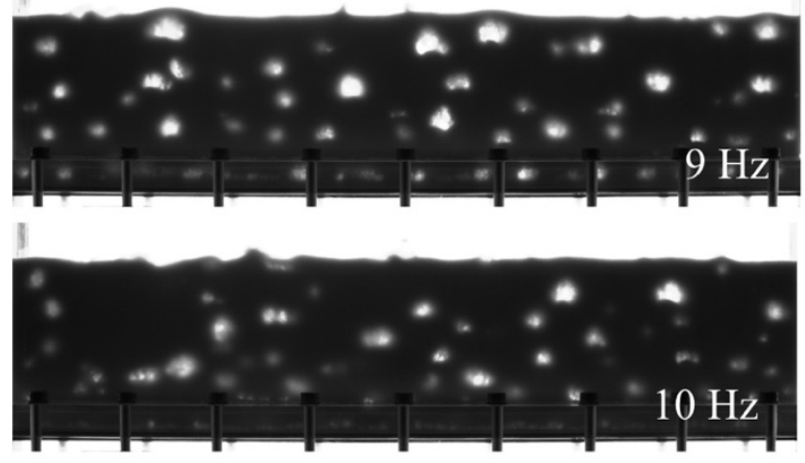

Figure 14: Example of unstructured bubble flow under pulsation. The particles are $238 \mu \mathrm{m}$ glass beads, with $u_{\mathrm{mf}}=4.1 \mathrm{~cm} / \mathrm{s}$. Bed height $h$ of $10 \mathrm{~cm}\left(420 D_{p}\right)$ and dimensionless gas superficial velocity $\hat{u}=u / u_{\mathrm{mf}}=0.5+1.75[1+\sin (2 \pi f t)]$ for (a) low $f=$ 3-4 Hz, (b) high $f=9-10 \mathrm{~Hz}$.

a Faraday instability, due to the complex flow involved in the rearrangement of the bubbles. Changes in $f$ and $u_{\mathrm{a}}$ condition the amount of air that is injected in every pulse and, with it, $D_{b}$ and $\lambda$. The average flowrate, $\bar{u}=u_{\min }+u_{a}$, on the other hand, determines the position of the oscillation relative to the minimum fluidization velocity, $u_{m f}$, and, hereby, the amount of gas that is injected in the bubbles or the emulsion phase (Figure 15). This appears to be a critical factor in the stability of a bubble pattern.

Oscillations where the superficial velocity of the gas, $u$, is maintained above the minimum fluidization velocity, $u_{m f}$, i.e., $\hat{u}_{\min }>1$, do not allow the propagation of a bubble pattern, at least in the study of monodispersed glass beads. It is necessary for the bed to temporarily defluidize. However, it is essential to realize that the system is inherently dynamic. Due to the persistent oscillation of the gas flow, it is constantly pushed out of equilibrium, creating simultaneous fluidlike like and solid-like zones at all times - thus, even if the velocity temporarily drops below $u_{m f}$, many particles remain in motion. Computational work $[51,198]$ and PTV (particle tracking velocimetry) analysis of ultrahigh speed videos [199] have associated the bubble self-organisation to the creation of intermittent locally dense areas in the bed that 
restrict the solid circulation. These areas form between each pair of bubbles where the solid flow converges, creating a jammed solid-like obstruction that prevents the lateral mixing. The stability of this pattern depends on the pulsation characteristics, $f, \hat{u}_{\min }$ and $\hat{u}_{\mathrm{a}}$, because they determine the relative size of the fluidization and defluidization cycles.
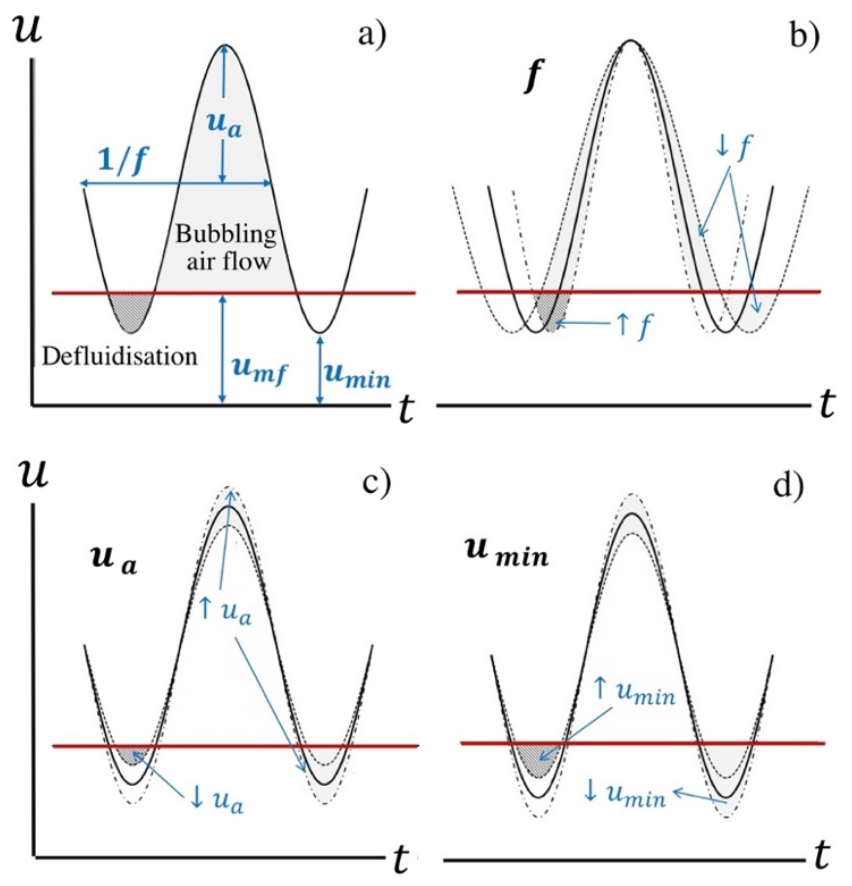

Figure 15: Schematic representation of the evolution of the gas superficial velocity $u$, during one and a half period of the gas pulsation, for a given minimum gas velocity, $u_{\text {min }}$, amplitude, $u_{a}$, and frequency, $f$; (a) When $u>u_{\mathrm{mf}}$, i.e., $\hat{u}>1$, gas is injected into the bubble phase, while there is partial defluidization during the time interval when $u<u_{\mathrm{mf}}$, i.e., $\hat{u}<1$. Depiction of the lengths of the time intervals and the quantities of gas in excess or in deficit with respect to $u_{\mathrm{mf}}$ with changes in (b) $f$, (c) $u_{a}$, and (d) $u_{\text {min }}$.

Figure 16 presents some examples of well-structured flows, including regular bubble patterns. Similar patterns can be obtained within a range of $f, \hat{u}_{\text {min }}$ and $\hat{u}_{\mathrm{a}}$ for glass beads of different sizes, ranging from 128 to $475 \mu \mathrm{m}$, but their stability varies. Some operating conditions lead to some spatial fluctuations in the initial bubble size, which occasionally causes bubbles to move laterally. This affects the motion of neighboring bubbles, and leads to overall variability in the pattern's structure, bubble size and velocity, but not to the point of causing coalescence or breakup, and distorting the pattern completely. Similarly, patterns become less stable higher up in the bed. 

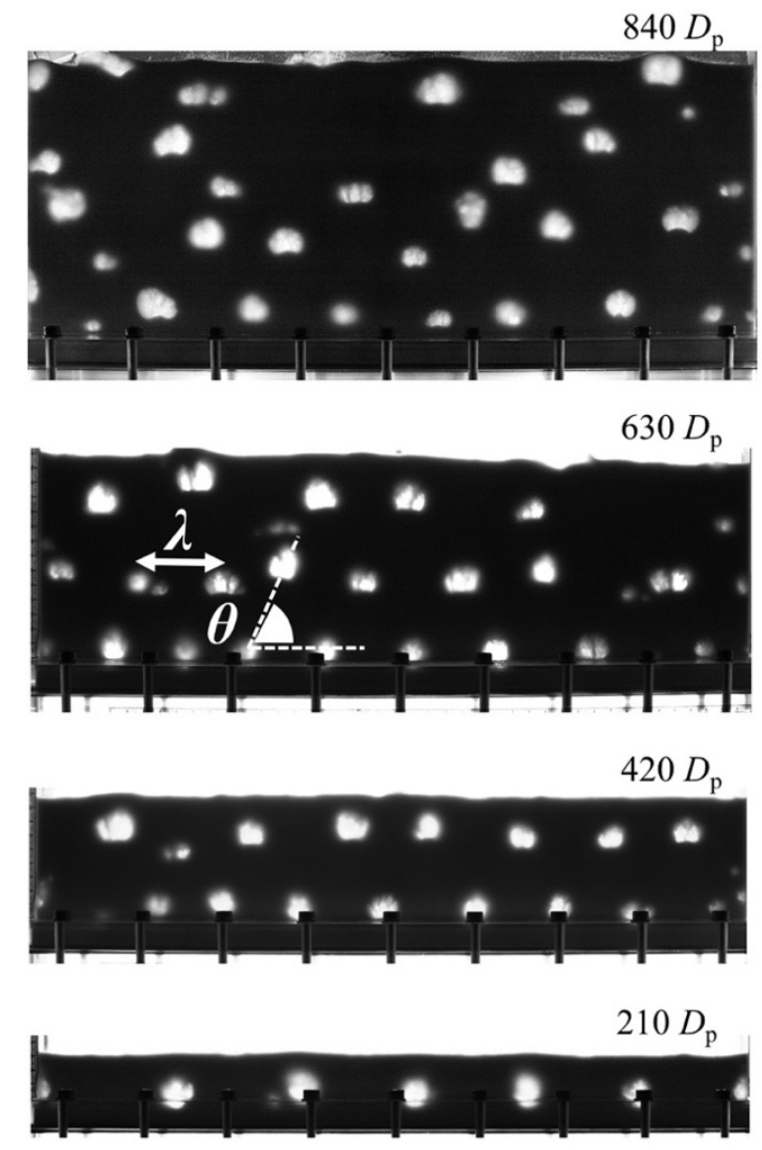

Figure 16: Example of a structured bubble flow under pulsation. The particles are $238 \mu \mathrm{m}$ glass beads, with $u_{\mathrm{mf}}=4.1 \mathrm{~cm} / \mathrm{s}$ and dimensionless gas superficial velocity $\hat{u}=u / u_{\mathrm{mf}}=0.5+1.75[1+\sin (2 \pi f t)]$. Bottom to top shows beds of increasing height $h$ of $5 \mathrm{~cm}\left(210 D_{p}\right), 10 \mathrm{~cm}\left(420 D_{p}\right), 15 \mathrm{~cm}\left(630 D_{p}\right), 20 \mathrm{~cm}\left(840 D_{p}\right)$.

Bubbles naturally grow and accelerate when they move up, so that the polar angle $\theta$ of the lattice increases axially (Figure 16). The relationship between $\lambda$ and $D_{b}$ shown in Figure 16 implies that there must be a specific inter-bubble distance and wavelength $\lambda$ associated to a horizontally aligned array of bubbles of size $D_{b}$. As $D_{b}$ increases in the upper part of the bed, bubbles find themselves arrayed with an "incorrect" wavelength, which is visible in the top image of Figure 16, and so they are forced to move laterally, introducing defects in the overall structure. Often, one bubble breaks and one of its daughters accelerates, moving diagonally upwards to a nodal position in a row above it. In the top region of a deep bed or away from the optimal conditions, these defects multiply, making the structure less reproducible and, eventually, indistinguishable from the operation of traditional operations, pulsed or not. 


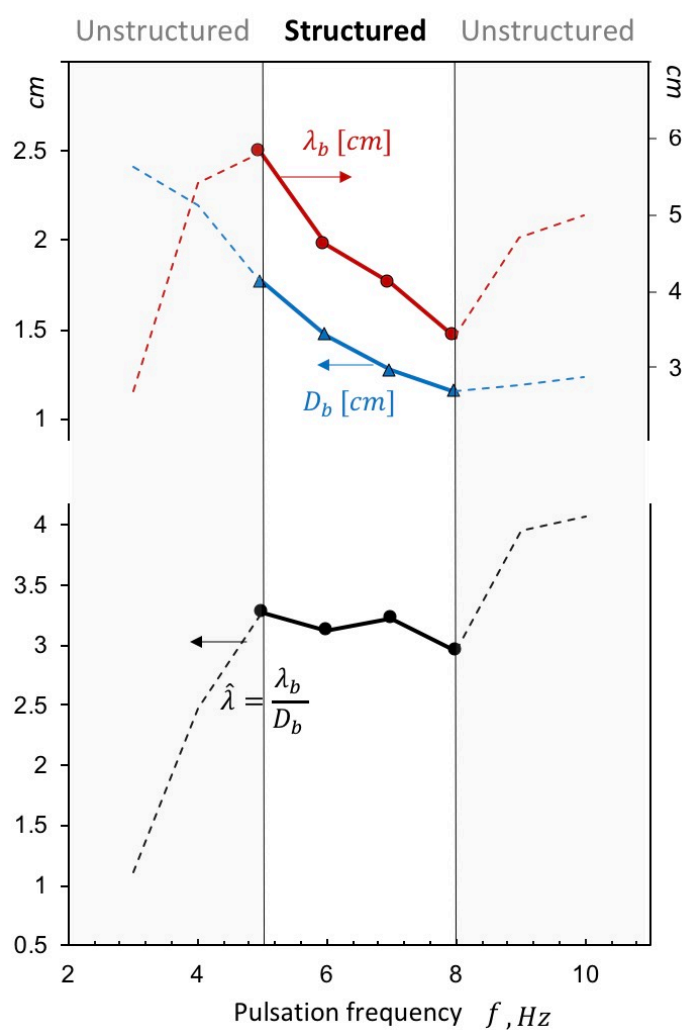

Figure 17: Manipulation of bubble size and wavelength via pulsation frequency. Bed height hof $10 \mathrm{~cm}$. The particles are $238 \mu \mathrm{m}$ glass beads, with $u_{\mathrm{mf}}=4.1 \mathrm{~cm} / \mathrm{s}$ and dimensionless gas superficial velocity $\hat{u}=u / u_{\mathrm{mf}}=0.5+1.75[1+\sin (2 \pi f t)]$.

Figure 17 illustrates the ability to control the bubble dynamics. It shows how the bubble size, $D_{b}$, and horizontal separation or wavelength, $\lambda_{b}$, evolve under conditions leading to structured and unstructured flows. For a given $u_{\mathrm{a}}, D_{b}$ decreases monotonically with increasing $f$ until it plateaus at high frequency in an unstructured operation $($ here, $f>8$ $\mathrm{Hz}$ ). In addition, at higher frequencies, the bubble size, $D_{b}$, becomes comparable to that observed in a fluidized bed at the same time-averaged, constant flow. Within the window of conditions leading to dynamic structuring ( 5 to $8 \mathrm{~Hz}$ ), the self-organization of the bubbles into a pattern establishes a correlation between $\lambda_{b}$ and $D_{b}$, forming a stable structure with an almost constant non-dimensional wavelength $\hat{\lambda}_{b}=\lambda_{b} / D_{b}$. In this way, under a structured operation one gains direct control over the bubble size and the separation, whereas, under unstructured pulsation, both vary independently and display a much broader distribution. 


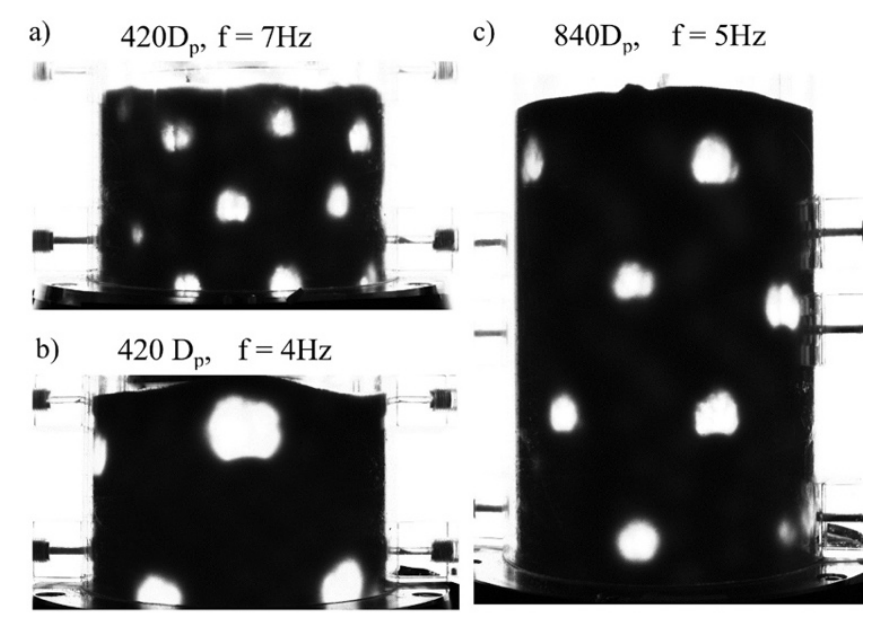

Figure 18: Example of a structured bubble flow under pulsation in an annular bed. The particles are $238 \mu \mathrm{m}$ glass beads, with $u_{\mathrm{mf}}$ $=4.1 \mathrm{~cm} / \mathrm{s}$ in beds of height $10 \mathrm{~cm}\left(420 D_{p}\right)(a$ and $b)$ and $20 \mathrm{~cm}\left(840 D_{p}\right)$, with a dimensionless gas superficial velocity of $(a) \hat{u}=$ $u / u_{\mathrm{mf}}=0.5+1.75[1+\sin (2 \pi 7 t)]$, (b) $\hat{u}=u / u_{\mathrm{mf}}=0.5+1.75[1+\sin (2 \pi 4 t)]$, and (c) $\hat{u}=u / u_{\mathrm{mf}}=0.5+1.75[1+$ $\sin (2 \pi 5 t)]$

It is worth emphasizing that the presence of the sidewalls does not support the pattern formation, contrary to linear standing waves, nor is $2 \mathrm{D}$ transport of the stress in the solid phase responsible for a pattern to emerge. In shallow beds, the pattern is independent of the container's shape - note the square pattern in Figure 5b, despite the cylindrical shape of the bed; this is an example of "symmetry breaking", typical for morphogenesis in nature [200]. Regular bubble patterns similar to the ones in Figure 16 could be produced experimentally in deep, annular, cylindrical beds, which amounts to having no sidewalls, but periodic boundaries (Figure 18). Despite the notable effects of curvature on the bubble nucleation and the stability of the pattern, a dynamically structured flow can be obtained up to, at least, a similar height as in a deep, flat vessel with a width equal to circumference of the annular bed.

By fine tuning the pulsation characteristics, it is possible to further extend the height up to which patterns persist in such structured beds. To do so, however, it is necessary to obtain a direct characterization of the solid flow patterns to understand the interaction between different bubble arrays. For practical applications, scale-up strategies must explore whether the formation of fully 3D patterns is possible. Such studies would include the effects of obstacles and the side boundaries to address the possibility of employing lateral and axial baffles to either stabilize a 3D pattern or otherwise design a collection of 2D patterns in a compartmentalized fashion. The dynamically structured beds described in this section sit somewhere between the operation of a fixed bed and a fluidized bed. They offer three main advantages. First, they can mix a powder operating at very low gas flowrate. Second, the pattern creates a compartmentalized flow, where the circulation of the solids is completely driven by the motion of adjoining bubble pairs. The structure decouples micromixing within each compartment, from long-range circulation or macromixing through the interaction of 
compartments [201]. Figure 19 illustrates the zones and the mixing patterns extracted from the analysis of CFD-DEM simulations. The entire solid phase follows the exact same array of circulation loops and, as a result, particle history is purely dominated by the scale of the pattern, that is, the bubble size and separation. Finally, in contrast to a typical pulsed bed, a dynamically structured bed is scalable and responsive. The scale of the pattern can be manipulated externally using the pulsation characteristics, and, in this way, define the solid circulation and the gas-contact pattern.

These three features open the way for alternative designs in a range of processes. Because mixing in dynamically structured beds can only be controlled at low slip velocities, heat and mass transport rates are likely to be below those in a traditional, vigorously bubbling fluidized bed. Therefore, dynamically structured units would not be advisable for systems currently operating at very large gas velocities, such as circulating fluidized beds. However, a dynamically structured unit will drastically reduce attrition, entrainment and the use of gas, while still ensuring local mixing, which can prove valuable in the intensification of processes involving high-value, toxic or dangerous particulate materials. In the context of reaction engineering, operation in a fixed bed allows for high slip velocities without entrainment, but the lack of particle mixing can lead to heterogeneity. A fluidized bed normally yields homogeneous mixing at the cost of attrition, entrainment and a lower conversion and selectivity. A dynamically structured bed would be advisable for systems sitting in between, where the use of gas and the loss of material must be minimized, but that still require a certain degree of mixing to ensure a homogeneous temperature and, critically, to reduce external transfer limitations by controlling sintering and agglomeration of the solids. In addition, the possibility to control solid mixing at low velocity can improve the energy efficiency of processes operating at low heat and mass transport rates, such as, for example, a solar irradiated fluidized bed collector, or lead to the design of new transport devices. The second feature, the creation of a very precise particle history (Figure 19), makes a dynamically structured bed of special interest for the intensification of particle formation units, such as dryers, mixers, coaters and agglomerators in the consumer goods industry, from pharmaceuticals to foods. The ability to establish a narrow particle stress distribution could prove to be the key to better control the dispersion and drying of binders, and particle breakage and growth processes. Finally, the responsive nature of these beds, illustrated in Figure 17, could allow for process integration, enabling the design of multistage processes, where the same unit can transition seamlessly across vastly different regimes. One can envisage the same unit moving from traditional operation at high velocity to maximize heat transfer at one stage (i.e. $1^{\text {st }}$ stage of drying), to an unstructured used of pulsation to maintain homogeneity and reduce attrition (i.e. $2^{\text {nd }}$ stage of drying when particles have become brittle) and to a dynamically structured operation to gain a tight control of solid mixing (i.e. coating or $3^{\text {rd }}$ stage of drying, when drying becomes dominated by internal diffusion). 
The possibility to control mixing is intimately related to the self-organization of the bubbles and the resulting solid circulation. This phenomenon is a direct consequence of the high solid fractions achieved during the pulsation. Sections of the bed in the wake of the bubbles and in between each pair of bubbles transition intermittently to a quasi-static granular flow, becoming jammed in frictional contact. In doing so, they create an obstacle that impedes the circulation

a)

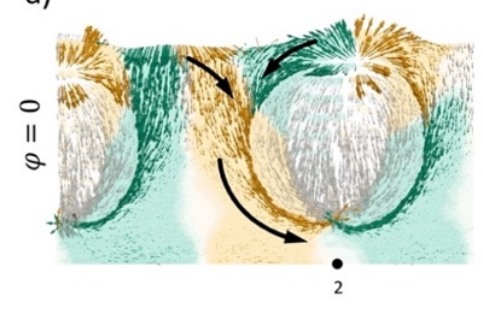

$$
\leftarrow \lambda / 2 \rightarrow
$$

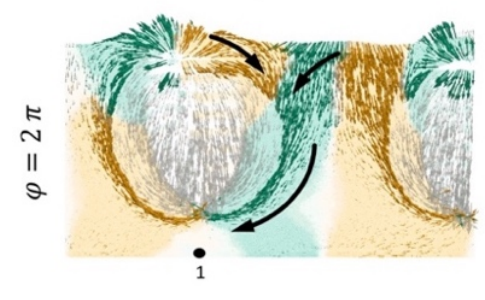

b)

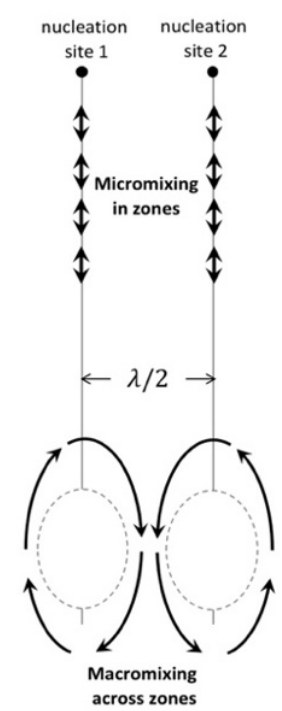

Figure 19: Solid flow compartmentalization. (a) CFD-DEM simulation [201]. Solid circulation in two consecutive pulses at phase angle $\varphi=0$ and $\varphi=2 \pi$. Vectors delimit the local solid velocity (white, scaled down $x 4$ ). Color marks the direction of lateral particle velocity, $v_{\mathrm{x}}\left(v_{\mathrm{x}}<0\right.$ green, $v_{\mathrm{x}}>0$ orange). (b) Resulting mixing patterns within the zone delimited by the bubble nucleation sites: particles mix axially within each zone, during each pulse, and zones interact within a fixed circulation loop.

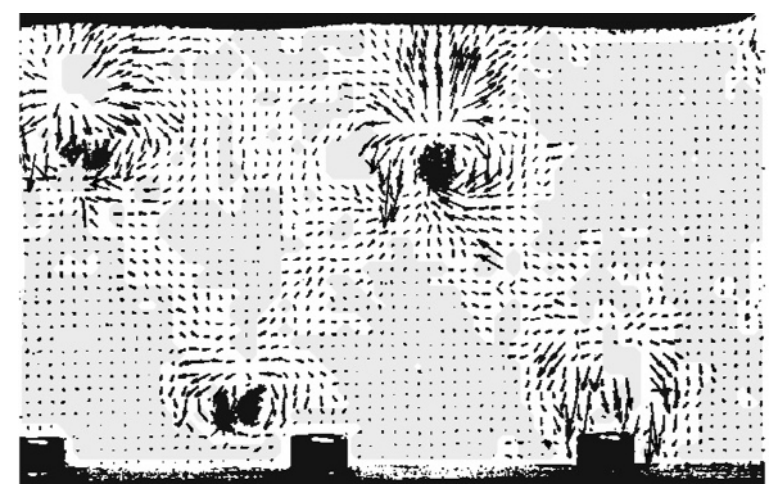

Figure 20: Experimental solid flow patterns in a pseudo-2D dynamically structured bed. Data from PTV analysis of high-speed videos. Arrows indicate the direction of the solid velocity. Areas of low velocity are shaded in grey. Bed height $h=10 \mathrm{~cm}$. The particles are $238 \mu \mathrm{m}$ glass beads, with $u_{\mathrm{mf}}=4.1 \mathrm{~cm} / \mathrm{s}$ and dimensionless gas superficial velocity $\hat{u}=u / u_{\mathrm{mf}}=0.5+1.25[1+$ $\sin (2 \pi 5 t)]$. 
of solids across the bed. These structures have been investigated in small systems, both experimentally, example in Fig 20, and computationally [198]. Studying the stability of these compartments when the pattern propagates upwards is a crucial step in future scale-up studies.

In quasi-2D beds, such as the ones described here, solid flow patterns can be studied with digital image analysis and well known PTV and PIV algorithms [199]. Figure 20 provides an example. Moving to larger 3D units will require the use of non-invasive tomographic systems, such as X-ray tomography [202, 203], electrical capacitance tomography (ECT) [204, 205] and capacitance volume tomography (ECVT) [206], or magnetic resonance imaging (MRI) [207, 208], perhaps in combination with optical fibre probes [209]. These are successful ways to study 3D bubbling dynamics at a relatively high time resolution, e.g., $100 \mathrm{~Hz}$. Tomographic systems do not possess a high (particle-scale) spatial resolution [210], but offer a sufficiently high level of detail to provide a good description of the overall emulsion phase concentration and velocity field, and identify the presence and, potentially, the size of these jammed areas.

The bubbling dynamics shown in Figures 13 and 16 is a scale independent phenomenon. It does not rely on the interaction with the side boundaries, but on the collective motion of the bubbles. It is worth flagging that this type of self-organization is not the only possible periodic flow structure induced by gas pulsation. Well known examples of patterns arising from the interaction with the boundaries in a shallow system include heaping or formation of concentric rings. Recently, the studies on pulsed bubbling by the National Energy Technology Laboratory [211, 212] discussed a similarly wall-dominated system and so-called "1-1" and "2-1" bubble configurations in a bed $5 \mathrm{~cm}$ wide and $0.5 \mathrm{~cm}$ thick using Geldart B particles and pulses of 5 to $6 \mathrm{~Hz}$. While in these works bubbling is reproducible, the bed operates under very low $\hat{u}_{\min }$ and very large $\hat{u}_{\mathrm{a}}$ where self-organization would not occur in a wider system. The reproducible structure described arises due to the effect of the side walls in the motion of the solids. In a bed not wide enough to accommodate the bubble pattern, self-organization is not allowed, and it is likely that no correlation exists between the operation conditions and the final bubble size and/or wavelength.

In summary, pulsation can be effectively used to alter the bubbling dynamics in a fluidized bed and create reproducible, scalable, macroscopic flows. Furthermore, in such dynamically structured fluidized beds, the size and separation of the bubbles can be externally controlled by changing the constant and pulsating components of the inlet gas flow. This provides an unprecedented level of control over the circulation in a gas-solid fluidized bed. In principle, it could allow using external actuation to bridge the behaviors associated to fixed and fluidized beds, separating micro- from macromixing. An example of dynamic self-organization, which is a universal structuring mechanism in natural systems, dynamically structured fluidized beds take advantage of the structuring induced by fluctuations in energy input and 
dissipation in a nonlinear system. This is very useful for practical applications, as the desired level of mixing between different species and the gas-solid contact time could now be tightly controlled, which is important in coating, drying, granulation or catalytic processes. Beyond the optimization and intensification of current operations, this may also open the way to entirely new application areas, benefitting from features of both fixed and fluidized beds.

\section{Theoretical challenges to simulate dynamically structured fluidized beds}

Substantial theoretical and computational progress has been made to simulate gas-solid fluidized beds using a multiscale approach, where different models represent the dominant physics at different scales [213-215]. Broadly speaking, continuum and discrete modeling approaches are employed. In a continuum framework, particle collisions are implicitly accounted for via the KTGF [216]. In discrete models, the equations of motions for the particles and their collisions are directly solved [217]. The previous sections showed how pattern formation in pulsed systems relies upon interphase friction and drag, as well as particle-level properties. The modeling and experimental studies of de Martín et al. [188, 190] suggest that interparticle friction does not influence the initiation of a pattern. The universal stability curve that characterizes the formation of the patterns in shallow layers does not depend on particle properties other than size. However, the conditions under which patterns propagate in bubbling fluidized beds are not the same, as this requires the bed to reach relatively dense conditions to mitigate the interference between bubbles and restrict long-range solid circulation. Modelling this phenomenon correctly requires not only accounting for a subtle spatiotemporal coupling between gas and solid phases, but also capturing transitions for the gas-solid suspension between dilute, inertial to dense, quasi-static flow, which remains an open fundamental question. When sustained multi-particle contacts are prevalent, the theoretical formulation of granular flows becomes much more challenging. This complication is exacerbated by the need to simultaneously model bubbles and other zones rich in gas, with low particle concentration.

Up to now, only few authors have tried to model oscillating fluidized beds. Van de Klundert [218] reported a first attempt, based on the one-dimensional Particle Array Model (PAM). The PAM is a highly simplified model for a fluidized bed, which describes the response of a vertical string of solid particles to a rising gas flow [191, 219]. In the absence of transversal motion, the authors reported how a string of 100 particles shows a chaos-to-order transition, to a highly ordered movement under a gas flow pulsing at frequencies, $f=5-15 \mathrm{~Hz}$. The most regular behavior was witnessed at $10 \mathrm{~Hz}$ for strings of 250 and 100 particles of $560 \mu \mathrm{m}$ diameter. A more comprehensive study was conducted by Kawaguchi et al. [220], who applied two-dimensional CFD-DEM simulations. The authors demonstrated that 
pulsation frequencies between $4-5 \mathrm{~Hz}$ allowed homogenizing the bubble flow in a $10 \mathrm{~cm}$ deep bed of Geldart B particles, but the pattern reported did not fully replicate the experimental observations. Two larger bubbles appeared simultaneously to align horizontally, but always at the same positions (Figure 21a). A few years later, Wang and Rhodes [221] reported that pulsing the gas at frequencies ranging from $5-10 \mathrm{~Hz}$ could "stabilize" the chaotic bubble flow of a $30 \mathrm{~cm}$ deep bed (Figure 21b). Once again, the nucleation sites in these simulations did not remain fixed, leading to an unstructured flow, different from experimental observations. In each of these cases, the nucleation of bubbles displayed some similarities with experiments, forming first a channel-like slug that spans horizontally at the bottom of the bed in each cycle and then breaks into bubbles, however, not in the correct positions.

a)
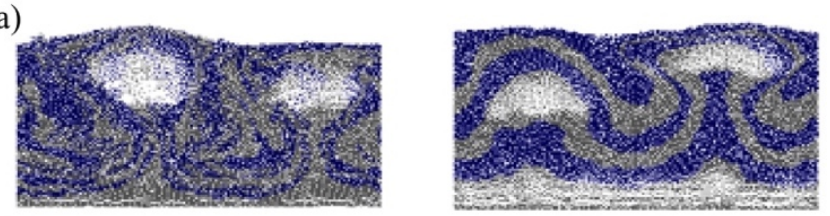

b)
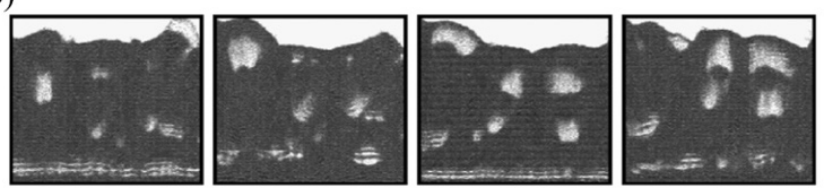

c)
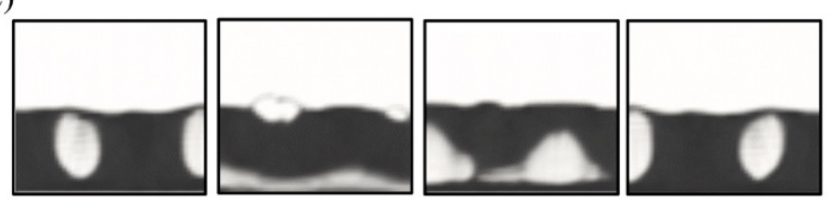

Figure 21: CFD-DEM models of oscillating fluidized beds. Snapshots of the solid flow pattern modelled by (a) Kawaguchi et al. [220], (b) Wang and Rhodes [221], (c) Wu et al. [51]. Reproduced with permission from [220, 221].

These early efforts concluded that pulsating the gas flow homogenizes the hydrodynamics, which aligns with common knowledge in pulsed fluidization. However, Section 4 demonstrates that oscillations can have much more profound effects, leading to a macroscopically structured bubble flow. Only recently, such phenomenon was reproduced numerically in a pulsation assisted bed using three-dimensional CFD-DEM simulations [51]. Simulations of $238 \mu \mathrm{m}$, Geldart B particles in a reduced quasi-2D domain ( $2 \mathrm{~mm}$ bed thickness) are able to replicate a subharmonic dynamic bubble pattern, stable in time and recurring at half of a pulsation frequency, $5 \mathrm{~Hz}$ (Figure 21c) Beyond the qualitative agreement with the experimentally observed bubbling flows, once a stable pattern is formed, quantitative agreement with experiments is obtained, where simulations accurately predict both bubble size and separation. The work shows that the bed alternates its behavior between fluid-like and solid-like. During the valley in each period of the sinusoidal signal, the bulk of the powder momentarily defluidizes, but the bubbles continue to rise toward the surface, which drives 
the flow of solids around them. The solids at the top of the bed are pushed towards the sides, colliding vigorously with each other, thus dissipating kinetic energy, create a dense area where they encounter the rising solids (central region of low particle velocity in Figure 22); however, the particles drawn from the space in between both rising bubbles at the lower section and into the bubble wakes concentrate and create a dense area that becomes almost stagnant on top of the distributor (lower left region of lower particle velocity in Figure 22).

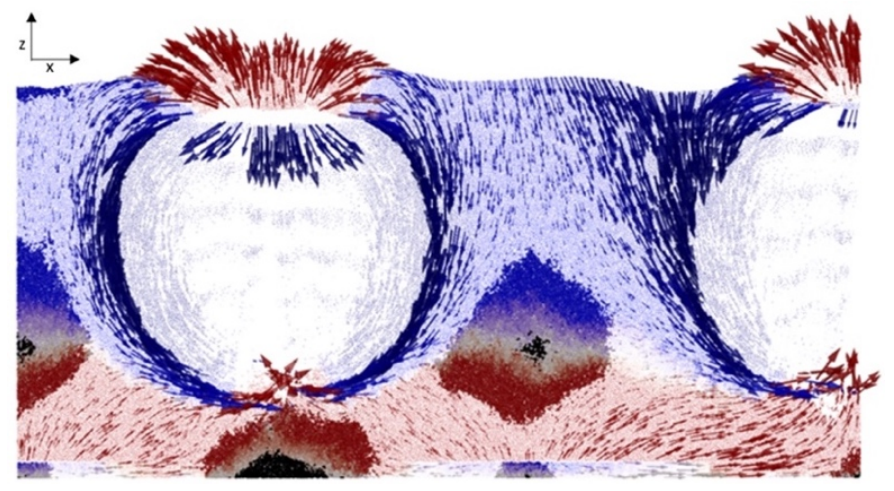

Figure 22: CFD-DEM of a structured flow. The areas of high non-dimensional particle velocity magnitude, $\hat{v}=v / d_{p}$, are marked with velocity vectors, for $\hat{v}>100$ (white arrows in the bubble, scaled down 4 times) and for $10<\hat{v}<100$ (colored arrows elsewhere). Areas of very low velocity are marked with particles for $1<\hat{v}<10$ (color), and $\hat{v}<1$ (black). The color of particles, arrows and background indicates the direction of the axial particle velocity, $v_{z}$ (blue: downwards, with $v_{z}<0$; white: $v_{z} \sim 0$, and red: upwards, with $v_{z}>0$ ). Particles are $238 \mu$ m glass beads under a dimensionless gas superficial velocity of $\hat{u}=u / u_{\mathrm{mf}}=0.5+$ $2.14[1+\sin (2 \pi 5 t)]$. The simulation domain shown is $10 \mathrm{~cm}$ wide by $4.5 \mathrm{~cm}$ high, and its thickness is $0.2 \mathrm{~cm}$.

In every pulse, a horizontal channel forms near the distributor and splits into bubbles when the gas velocity starts to decrease. The breakage process is then conditioned by the stresses caused by the wakes of the preceding array of bubbles. The pyramid-shaped solid regions in the wake of each bubble shown in Figure 22 are consolidated by the solid circulation induced by the array of bubbles in front. The solid flow creates a downwards compressive stress causing the presence of the bubble to exert an additional pressure onto the distributor plate. This resistance counters the drag force exerted by the fluid, so that the gas slug created in the following pulse tends to accumulate gas in the spaces left in between the dense areas left behind the previous bubbles' wakes, and in this way forms a new set of bubbles staggered in between the previous ones.

Provided the bubbles formed are of the same size, the dense areas created in their wakes are equally spaced, which eventually leads to two possible sets of nucleation sites that alternate in every pulse to form the observed pattern. Large bubbles are robust and rise fast, causing a rapid solid circulation in their proximity and large dense regions in their wake. Therefore, they stabilize in a pattern of a large wavelength, whereas small bubbles drive less solid around them and, 
therefore, become closely spaced, which makes them more sensitive to unbalance. This may explain why, at large frequencies, the pattern destabilizes (Figure 14b).

Frictional stress is essential to describe bubbling in a fluidized bed [222]. In a pulsed bed, its effect is only exacerbated, because the granular suspension is compressed and re-fluidized periodically. Recent numerical work conducted by the National Energy Technology Laboratory and MIT [211] on a smaller pulsed bed associates a coherent flow structures with a high level of energy dissipation, due to friction and inelasticity. As mentioned earlier, the creation of so-called "1-1" and "2-1" bubbling in a small bed ( $5 \mathrm{~cm}$ wide and $0.5 \mathrm{~cm}$ thick), is dominated by the interaction with the boundaries, whereas larger systems are driven by the effect of the collective motion of the bubbles on the solid flow. De Oliveira et al. also studied the patterns formed in pulsed beds of $16 \mathrm{~cm}$ wide and $0.2 \mathrm{~cm}$ thick, using CFD-DEM simulations [225]. They observed time-averaged pressure drop and coordination number peaks around the pulse condition of a non-dimensional minimum velocity of $\hat{u}_{\min } \sim 1\left(u_{\min } \sim u_{\mathrm{mf}}\right)$, which is indicative of sustained contacts in the solid phase. As already indicated by $\mathrm{Wu}$ et al. [51], frictional properties and energy dissipation will undoubtedly play an important role in the self-organization of bubbles in fluidized beds. For that reason, continuum models based

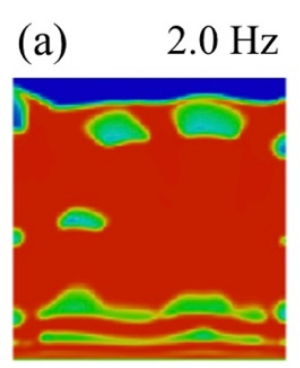

(b) $\quad 2.5 \mathrm{~Hz}$
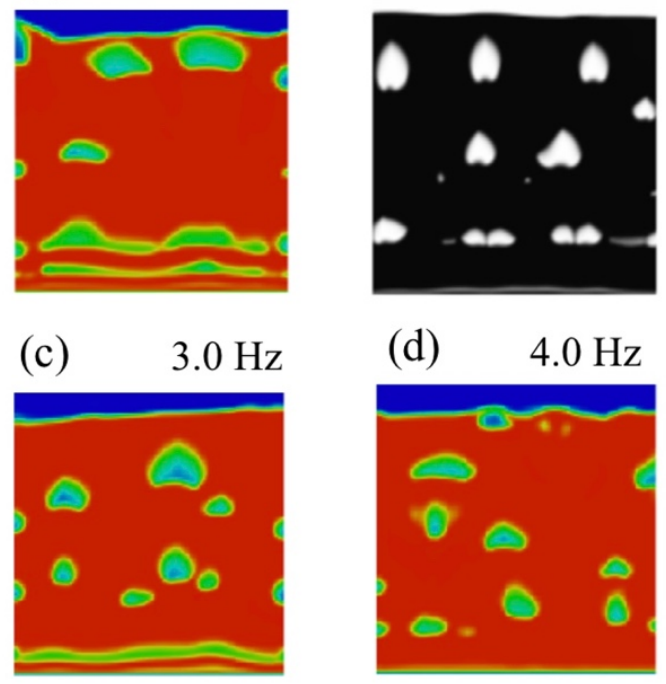

Figure 23: TFM simulations of pulsed gas-solid fluidized beds of $360 \mu \mathrm{m}$ sand particles, with a static bed height of $40 \mathrm{~cm}$ and $u_{\mathrm{mf}}=$ $0.13 \mathrm{~m} / \mathrm{s}$. Snapshots of the void fraction from $(a, c, d) 2 D$ simulations, by Wu et al. [223] with a dimensionless gas superficial velocity of $\hat{u}=u / u_{\mathrm{mf}}=1.0+0.4[1+\sin (2 \pi f t)]$ with $f=2,3$ and $4 \mathrm{~Hz}$. (b) $3 D$ simulations, by Bakshi et al. [224] with a nondimensional gas superficial velocity of $\hat{u}=u / u_{\mathrm{mf}}=1.0+0.4[1+\sin (2 \pi 2.5 t)]$. Reproduced with permission from the references given. 
on the KTGF have not yet been able to replicate the same behavior convincingly [199]. Even integrating frictional stress models underpinned by the critical-state theory of soil mechanics seem to lack a sufficiently accurate description of the physics to reproduce complex gas-driven patterns.

2D TFM simulations [223] have shown that a square pattern forms occasionally, but it is unstable. Bakshi et al. have recently studied 3D domains with continuum models [224]. The authors report important differences in the bubbling behavior, due to the effects of the walls on the energy dissipation (Figure 23b). 3D simulations provide a more reproducible bubbling dynamic at $f=4 \mathrm{~Hz}$, and, according to the authors, a more structured arrangement characterized by horizontal arrays. However, such bubble patterns are also intermittent and do not agree with the experimental observation of a triangular lattice (i.e., staggered bubble arrays with alternating bubble positions), and instead show a similar square lattice (i.e., aligned bubble arrays with fixed bubble positions) behavior to the 2D TFM simulations reported in [223]. Since the resulting structure in a 3D system does not replicate the experimental pattern, it must be ascribed to limitations of the model's underlying physics, whereby some kind of pattern arises as a numerical artefact.

The inability of any continuum modelling framework, hitherto, to describe the phenomenon of dynamically structured fluidized beds is unsurprising, due to its reliance on the KTGF, which cannot be expected to hold for dense flows. Its formulation is based upon binary and instantaneous collisions of frictionless particles. Therefore, it does not account for stresses due to enduring contacts or the effects of mesoscopic structures and correlated velocity distributions arising in the transition towards a quasi-static regime [226, 227]. The underpredicted stress is commonly corrected by the introduction of an added frictional contribution. Due to its empirical (or sometimes semi-empirical) nature, the solid pressure term takes various functional forms, depending on the authors, and can vary in its predictions over 10 orders of magnitude (Figure 24a). Therefore, the resulting dense solid phases in a TFM framework are extremely sensitive to the model employed to compute frictional stress and to the frictional packing limit, $\varepsilon_{s, \mathrm{f}}$, above which the frictional stress is applied (Figure 24b) [228]. Increasing $\varepsilon_{\mathrm{s}, \mathrm{f}}$ delays the onset of frictional effects, which allows the emulsion phase to contract to a higher extent, while less gas bypasses through the emulsion phase, but forms more bubbles. At low $\varepsilon_{\mathrm{s}, \mathrm{f}}$, the solid phase experiences increasing repulsive solid pressure when it contracts, and, then, it remains in a relatively dilated state. Therefore, more gas flows into the emulsion phase to maintain its suspended state, and the injected gas initially forms a horizontal slug across the bed, without breaking into voids. Nevertheless, such a sustained state would be unphysical. By increasing $\varepsilon_{\mathrm{s}, \mathrm{f}}$ to an intermediate value, e.g., $\varepsilon_{\mathrm{s}, \mathrm{f}}=0.55$, the slug breaks after detaching from the distributor into two large bubbles during each cycle. As described before, these two bubbles always nucleate at fixed positions and rise in line. This flow pattern is very stable and repeats itself periodically, but it does not represent the experimental 
pattern, which again must be attributed to a numerical artefact. When $\varepsilon_{\mathrm{s}, \mathrm{f}}$ further increases, the simulated flow pattern becomes unstable, and bubbling progresses in a similar way to the observations in fluidized beds with a constant inlet flow.

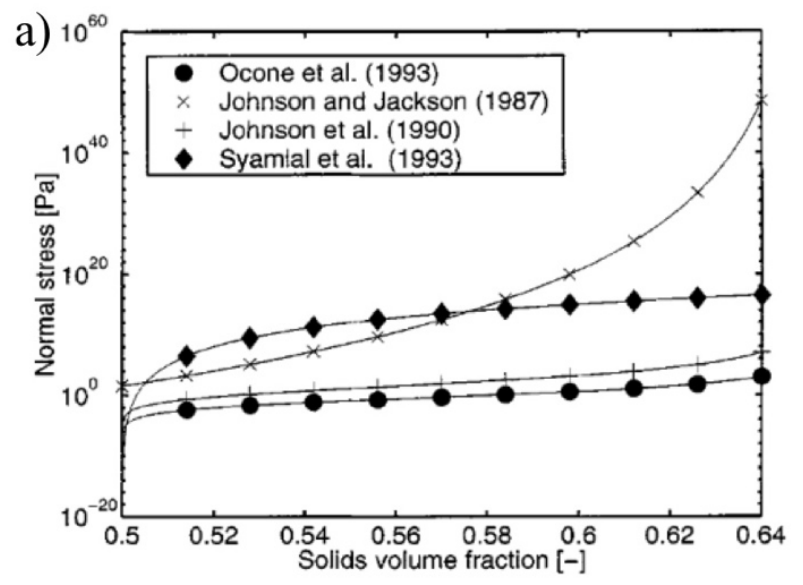

b)

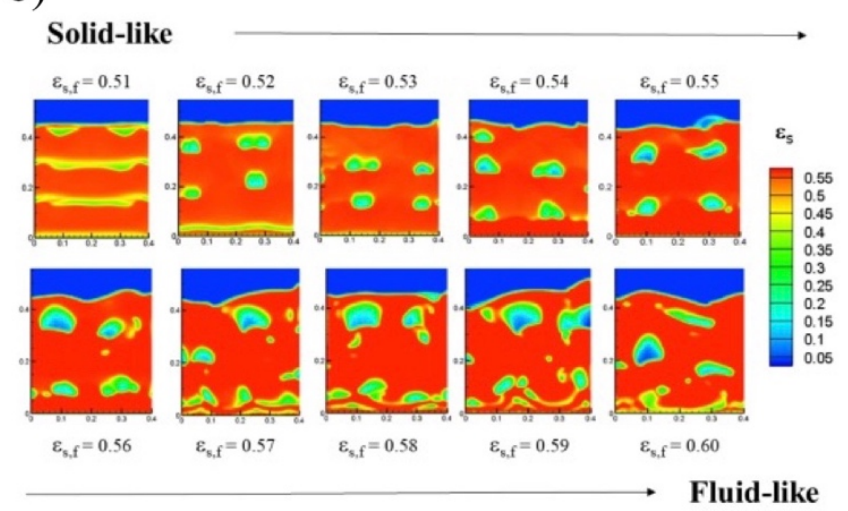

Figure 24: (a) Range of predictions of classic solid frictional models. Reproduced with permission from [228], comparing models from [193, 229, 230]. (b) Illustration of the effect of the frictional packing limit, $\varepsilon_{s}$, , in a pulsed fluidized bed. Void fraction plots for increasing Es,f, using Schaeffer's expression for the frictional viscosity [231], the Johnson-Jackson expression for frictional pressure [229], and Mazzei and Lettieri's expression for the drag [232]. Simulations carried out for $360 \mu \mathrm{m}$ glass beads with $u_{\mathrm{mf}}=$ $0.13 \mathrm{~m} / \mathrm{s}$ in a bed of height $h=40 \mathrm{~cm}\left(\sim 1110 D_{p}\right)$ and non-dimensional gas superficial velocity of $\hat{u}=u / u_{\mathrm{mf}}=1.0+0.4[1+$ $\sin (2 \pi 4 t)]$

Over the last few years, there has been increasing awareness of the deficiencies of critical-state based models and a demand for predicting dense granular flow behavior using coarse-scale approaches for large units, which remain infeasible to model by a discrete, CFD-DEM approach, due to the computationally prohibitive number of particles [233]. Tremendous effort has been devoted to new, macroscopic descriptions that capture the dense flow rheology and effects of interparticle friction. These works extend the existing KTGF in the Eulerian framework to account for the impact of interparticle friction in the dense, quasi-static regime, by including effective restitution coefficients [234-236], through 
"mesoscience" by addressing also interactions at the mesoscale in variational approaches [237, 238], introducing rotational granular energy and an associated effective viscosity [239, 240], and constructing granular flow localrheology [226, 241-243] and non-local rheology laws [244-246] that govern the correlation between stress and strain rate. These promising methods provide a theoretical basis for future development and have been discussed in more detail in a recent work [199].

\section{Conclusions and future steps}

Knowledge on how spatial patterns are initiated and propagate in granular media has matured enough over the last few decades to create them reliably, experimentally in the laboratory. However, the fundamental mechanisms underpinning these phenomena in mechanical and gas-driven systems are still the subject of debate. Studies of vertically vibrated granular layers point to different conclusions regarding the role of friction in the stabilization of surface patterns [169, $177,180,185]$, associating its role to specific features in the surface waves, rather than to the instability itself. Pulsing the gas flow or vibrating the bed at a high frequencies, above $9 \mathrm{~Hz}$, leads to a relatively dilute state, where frictional stresses are less pronounced [180, 247]. However, when the flow is pulsed at low frequencies, 3 to $7 \mathrm{~Hz}$, even though similar patterns emerge, they do so in a dense environment, where different physical laws must be considered. In shallow layers, the friction between solid particles seems to play virtually no role on the formation of surface wave patterns, according to the experiments of de Martín et al. [188], where the universal onset criterium is independent of the particle properties. Up to this point, the initiation of surface waves is a purely hydrodynamic manifestation of the system. However, a correct description of frictional contacts seems crucial to reproduce the propagation of a wave into a macroscopic bubbling pattern in deep beds. Comparison of experiments with computational works based on discrete models establishes that the emulsion phase must be dense enough for bubbles to self-organize into a pattern, and the failure of two-fluid model formulations to replicate this phenomenon indicates that a faithful representation of solid stresses, especially of the frictional contribution that bridges the kinetic and the plastic regimes, is still missing in the classic formulations.

There is significant progress in our ability to control granular patterns. Nevertheless, more work is required to create and control flow structures at sufficiently large scale for practical use in a reproducible manner. In order to exploit dynamically structured fluidization for the purpose of both process intensification and advanced fundamental understanding of gas-solid mechanics, the following four areas for future areas of work are encouraged: 
Stability: Macroscopic patterns correspond to the organization of gas bubbles into a specific arrangement. Different from a surface wave, such structures can admit the presence of defects, which leads to different degrees of stability, not only across operation conditions but also spatially (patterns degrade as bubbles move up) and temporally (patterns can break up and then reconstruct periodically). As a result, large-scale ordered flows emerge under a wide range of conditions with a different degree of order or stability. It is necessary to move onto rigorous ways of describing quantitatively the degree of order in the bubble dynamics, rather than the somewhat subjective, visual assignment of what constitutes an "ordered pattern". This will unlock the identification of a universal operation regime for dynamically structured bubbling beds, as well as facilitate the study of its underpinning physics and the operational benefits associated to the creation of a pattern. Along these lines, it is important to investigate the role that the energy dissipated through frictional contacts plays in the bubble nucleation and propagation of patterns, from both a theoretical and an experimental perspective. That entails studying other important aspects of the solids, such as the interparticle friction coefficient, particle restitution coefficient, stiffness, shape, polydispersity, and the gas phase properties, such as density gradients induced by reactive processes.

Scale-up: In order to broaden the applicability of structured flows, it is imperative to learn ways to scale up the macroscopic bubble pattern observed in quasi-2D systems to fully 3D structures. Analysis of the stability of 3D patterns and their interaction with the walls or potential internals requires a better understanding of the solid flow. It is important to conduct numerical and experimental work based on PIV to elucidate how different bubble arrays interact laterally and axially in 2D patterns. The effects of the curvature of the walls in a deep cylindrical bed are worthy of further investigation. In particular, a compelling opportunity for scale-up is the use of quasi-2D, annular beds (Figure 18). These could be seen as a first step toward multi-annular systems or multi-rectangular systems, along with the use of baffles and internals to stabilize a 3D flow. For fully 3D systems, the assistance of non-invasive tomographic techniques, such as ECT and X-ray tomography, as well as MRI, would provide valuable insights.

Transfer coefficients: For any study on the performance of dynamically structured fluidized beds, it is essential to investigate how solid mixing and bubble patterns affect the contact time between the gas and the solids, and the overall mass and heat transfer coefficients. This influences how gas-solid and heterogeneously catalyzed reactions, particle coating, drying and granulation processes are affected, and how their performance can be improved by dynamic structuring. 
Modelling strategies: From a theoretical perspective, important efforts have been devoted to improving how friction is accounted for in dense granular flows. Advanced frictional stress models and modified formulations of the KTGF are being developed to describe the transition between the viscous and the plastic regime [226, 242]. These are very promising steps, but more progress is needed, and they are not yet widely applied to industrial multiphase flows. Pattern formation in oscillating fluidized beds represents a new, tough dynamic benchmark for any such models, and they may provide a pathway for developing new closures to merge the dilute and dense regimes. From a practical standpoint, studying the performance of coarse-grained, discrete approaches and hybrid models is important to create a platform that allows the study of large dynamically structured fluidized beds for applications across different industries.

In summary, research over the last few decades has demonstrated that it is possible to create dynamically structured fluidized beds using periodic gas flow oscillations to stabilize the inherently chaotic hydrodynamics. The attractive result is a directly scalable and fully predictable flow. This provides tight control over solids mixing and gas-solid contact time, promising process performance that borrows advantages from both fixed and fluidized beds with constant inlet gas flow. Significant scale-up work lies ahead, but this approach represents a new avenue for the intensified design of gas-solid operations - not only the optimization of existing processes, but also the creation of new designs and operations. This is based on the ability to externally fine-tune a dynamic flow structure, in the same way that hierarchical structuring has transformed fixed bed reactors and catalysts, but now using the power of dynamics as a self-organizing principle in nature.

\section{Acknowledgements}

The research leading to these results has received funding from an EPSRC "Frontier Engineering" Award (EP/K038656/1) and an EPSRC Doctoral Training Award (1528604).

\section{References}

[1] L.-S. Fan, C. Zhu, Principles of gas-solid flows, Cambridge University Press, Cambridge, 2005.

[2] M.J. Rhodes, Introduction to particle technology, John Wiley \& Sons, New Jersey, 2008.

[3] R. Cocco, S.R. Karri, T. Knowlton, Introduction to fluidization, CEP 110 (2014) 21-29.

[4] J.P.K. Seville, C.D. Willett, P.C. Knight, Interparticle forces in fluidisation: a review, Powder Technol. 113 (2000) 261-268. 
[5] J. Shabanian, J. Chaouki, Local characterization of a gas-solid fluidized bed in the presence of thermally induced interparticle forces, Chem. Eng. Sci. 119 (2014) 261-273.

[6] L.G. Gibilaro, Fluidization dynamics, Butterworth-Heinemann, Oxford, 2001.

[7] M. Rüdisüli, T.J. Schildhauer, S.M.A. Biollaz, J.R. van Ommen, Scale-up of bubbling fluidized bed reactors — A review, Powder Technol. 217 (2012) 21-38.

[8] L.R. Glicksman, M. Hyre, K. Woloshun, Simplified scaling relationships for fluidized beds, Powder Technol. 77 (1993) 177199.

[9] J.G. Yates, P. Lettieri, Fluidized-bed scaling, in: Fluidized-bed reactors: processes and operating conditions, Springer, Cham, 2016, pp. 175-194.

[10] C.S. Daw, C.E. Finney, M. Vasudevan, N.A. Van Goor, K. Nguyen, D.D. Bruns, E.J. Kostelich, C. Grebogi, E. Ott, J.A. Yorke, Self-organization and chaos in a fluidized bed, Phys. Rev. Lett. 75 (1995) 2308.

[11] C.M. van den Bleek, M.-O. Coppens, J.C. Schouten, Application of chaos analysis to multiphase reactors, Chem. Eng. Sci. 57 (2002) 4763-4778.

[12] C.M. van den Bleek, J.C. Schouten, Deterministic chaos: a new tool in fluidized bed design and operation, Chem. Eng. J. and Biochem. Eng. J. 53 (1993) 75-87.

[13] J.R. van Ommen, S. Sasic, J. van der Schaaf, S. Gheorghiu, F. Johnsson, M.-O. Coppens, Time-series analysis of pressure fluctuations in gas-solid fluidized beds - A review, Int. J. Multiphase Flow 37 (2011) 403-428.

[14] J.G. Yates, Fundamentals of fluidized-bed chemical processes, Butterworth-Heinemann, Boston, 2013.

[15] N. Kobayashi, R. Yamazaki, S. Mori, A study on the behavior of bubbles and solids in bubbling fluidized beds, Powder Technol. 113 (2000) 327-344.

[16] D. Kunii, O. Levenspiel, Fluidization engineering, Butterworth-Heinemann, Boston, 2013.

[17] J.R. Grace, Hydrodynamics of Bubbling Fluidization, in: Essentials of Fluidization Technology, Wiley-VCH, Weinheim, 2020, pp. 131-152.

[18] W. Zhang, A review of techniques for the process intensification of fluidized bed reactors, Chin. J. Chem. Eng. 17 (2009) $688-702$.

[19] F. Raganati, R. Chirone, P. Ammendola, Gas-solid fluidization of cohesive powders, Chem. Eng. Res. Des. 133 (2018) $347-$ 387.

[20] L. Song, T. Zhou, J. Yang, Fluidization behavior of nano-particles by adding coarse particles, Adv. Powder Technol. 20 (2009) 366-370.

[21] G.C. Brouwer, E.C. Wagner, J.R. van Ommen, R.F. Mudde, Effects of pressure and fines content on bubble diameter in a fluidized bed studied using fast X-ray tomography, Chem. Eng. J. 207-208 (2012) 711-717. 
[22] L.J. McLaughlin, M.J. Rhodes, Prediction of fluidized bed behaviour in the presence of liquid bridges, Powder Technol. 114 (2001) 213-223.

[23] C.M. Boyce, A. Penn, M. Lehnert, K.P. Pruessmann, C.R. Müller, Effect of liquid bridging on bubbles injected into a fluidized bed: A magnetic resonance imaging study, Powder Technol. 343 (2019) 813-820.

[24] K. Rietema, H.W. Piepers, The effect of interparticle forces on the stability of gas-fluidized beds-I. Experimental evidence, Chem. Eng. Sci. 45 (1990) 1627-1639.

[25] J. Shabanian, J. Chaouki, Effects of temperature, pressure, and interparticle forces on the hydrodynamics of a gas-solid fluidized bed, Chem. Eng. J. 313 (2017) 580-590.

[26] J.M. Valverde, M.A.S. Quintanilla, A. Castellanos, D. Lepek, J. Quevedo, R.N. Dave, R. Pfeffer, Fluidization of fine and ultrafine particles using nitrogen and neon as fluidizing gases, AlChE J. 54 (2008) 86-103.

[27] D.C. Chitester, R.M. Kornosky, L.-S. Fan, J.P. Danko, Characteristics of fluidization at high pressure, Chem. Eng. Sci. 39 (1984) 253-261.

[28] H. Li, W. Yan, W. Wu, C. Wang, Characteristics of fluidisation behaviour in a pressurised bubbling fluidised bed, Can. J. Chem. Eng. 91 (2013) 760-769.

[29] F. Niu, B. Subramaniam, Particle fluidization with supercritical carbon dioxide: experiments and theory, Ind. Eng. Chem. Res. 46 (2007) 3153-3156.

[30] V. Martín, M.J. Cocero, S. Rodríguez-Rojo, Fluidization of nanoparticles agglomerates enhanced by supercritical carbon dioxide, Powder Technol. 318 (2017) 242-247.

[31] P. Azadi, R. Farnood, Review of heterogeneous catalysts for sub- and supercritical water gasification of biomass and wastes, Int. J. Hydrogen Energ. 36 (2011) 9529-9541.

[32] J. Huang, Y. Lu, H. Wang, Fluidization of particles in supercritical water: a comprehensive study on bubble hydrodynamics, Ind. Eng. Chem. Res. 58 (2019) 2036-2051.

[33] C. Tregambi, R. Chirone, F. Montagnaro, P. Salatino, R. Solimene, Heat transfer in directly irradiated fluidized beds, Sol. Energy 129 (2016) 85-100.

[34] S. Mehran, M. Nikian, M. Ghazi, H. Zareiforoush, I. Bagheri, Experimental investigation and energy analysis of a solarassisted fluidized-bed dryer including solar water heater and solar-powered infrared lamp for paddy grains drying, Sol. Energy 190 (2019) 167-184.

[35] F. Mushtaq, R. Mat, F.N. Ani, A review on microwave assisted pyrolysis of coal and biomass for fuel production, Renew. Sust. Energ. Rev. 39 (2014) 555-574.

[36] R. Jumah, Modelling and imulation of continuous and intermittent radio frequency-assisted fluidized bed drying of grains, Food Bioprod. Process. 83 (2005) 203-210. 
[37] X. Zhou, S. Wang, Recent developments in radio frequency drying of food and agricultural products: A review, Drying Technol. 37 (2019) 271-286.

[38] C. Si, J. Wu, Y. Wang, Y. Zhang, G. Liu, Effect of acoustic field on minimum fluidization velocity and drying characteristics of lignite in a fluidized bed, Fuel Process. Technol. 135 (2015) 112-118.

[39] Q. Guo, H. Liu, W. Shen, X. Yan, R. Jia, Influence of sound wave characteristics on fluidization behaviors of ultrafine particles, Chem. Eng. J. 119 (2006) 1-9.

[40] J.R. van Ommen, J. Nijenhuis, M.-O. Coppens, Reshaping the structure of fluidized beds, CEP (2009) 49-57.

[41] R. Cocco, A. Issangya, S.B.R. Karri, T. Freeman, H.M. Jaeger, T.M. Knowlton, Small-scale particle interactions are having significant effects on global fluidized bed behavior, KONA Powder Part. J. 34 (2017) 155-167.

[42] A. Anantharaman, R.A. Cocco, J.W. Chew, Evaluation of correlations for minimum fluidization velocity (Umf) in gas-solid fluidization, Powder Technol. 323 (2018) 454-485.

[43] Y. Shao, W. Zhong, A. Yu, Mixing behavior of binary and multi-component mixtures of particles in waste fluidized beds, Powder Technol. 304 (2016) 73-80.

[44] C.E. Agu, C. Pfeifer, B.M.E. Moldestad, Prediction of void fraction and minimum fluidization velocity of a binary mixture of particles: Bed material and fuel particles, Powder Technol. 349 (2019) 99-107.

[45] M. Rasteh, F. Farhadi, G. Ahmadi, Empirical models for minimum fluidization velocity of particles with different size distribution in tapered fluidized beds, Powder Technol. 338 (2018) 563-575.

[46] Y. Shao, Z. Li, W. Zhong, Z. Bian, A. Yu, Minimum fluidization velocity of particles with different size distributions at elevated pressures and temperatures, Chem. Eng. Sci. 216 (2020) 115555.

[47] G. Sun, J. Grace, Effect of particle size distribution in different fluidization regimes, AlChE J. 38 (1992) 716-722.

[48] J. Grace, G. Sun, Fines concentration in voids in fluidized beds, Powder Technol. 62 (1990) 203-205.

[49] A. Ajbar, K. Alhumazi, M. Asif, Improvement of the fluidizability of cohesive powders through mixing with small proportions of group A particles, Can. J. Chem. Eng. 83 (2005) 930-943.

[50] D. Jia, X. Bi, C.J. Lim, S. Sokhansanj, A. Tsutsumi, Gas-solid mixing and mass transfer in a tapered fluidized bed of biomass with pulsed gas flow, Powder Technol. 316 (2017) 373-387.

[51] K. Wu, L. de Martín, M.-O. Coppens, Pattern formation in pulsed gas-solid fluidized beds-the role of granular solid mechanics, Chem. Eng. J. 329 (2017) 4-14.

[52] J. Baeyens, D. Geldart, S.Y. Wu, Elutriation of fines from gas fluidized beds of Geldart A-type powders — effect of adding superfines, Powder Technol. 71 (1992) 71-80.

[53] S. Maurer, E.C. Wagner, J.R. van Ommen, T.J. Schildhauer, S.L. Teske, S.M.A. Biollaz, A. Wokaun, R.F. Mudde, Influence of vertical internals on a bubbling fluidized bed characterized by X-ray tomography, Int. J. Multiphase Flow 75 (2015) $237-249$. 
[54] S. Yang, H. Li, Q. Zhu, Experimental study and numerical simulation of baffled bubbling fluidized beds with Geldart A particles in three dimensions, Chem. Eng. J. 259 (2015) 338-347.

[55] C.N. Lim, M.A. Gilbertson, A.J.L. Harrison, Characterisation and control of bubbling behaviour in gas-solid fluidised beds, Control Eng. Pract. 17 (2009) 67-79.

[56] C.A.M. Silva, M.R. Parise, F.V. Silva, O.P. Taranto, Control of fluidized bed coating particles using Gaussian spectral pressure distribution, Powder Technol. 212 (2011) 445-458.

[57] M.-O. Coppens, J.R. van Ommen, Structuring chaotic fluidized beds, Chem. Eng. J. 96 (2003) 117-124.

[58] D. Christensen, J. Nijenhuis, J.R. van Ommen, M.-O. Coppens, Residence times in fluidized beds with secondary gas injection, Powder Technol. 180 (2008) 321-331.

[59] J.M. Valverde, A. Castellanos, Fluidization of nanoparticles: A simple equation for estimating the size of agglomerates, Chem. Eng. J. 140 (2008) 296-304.

[60] F. Raganati, R. Chirone, P. Ammendola, Calcium-looping for thermochemical energy storage in concentrating solar power applications: Evaluation of the effect of acoustic perturbation on the fluidized bed carbonation, Chem. Eng. J. 392 (2020) 123658. [61] J. De Wilde, Gas-solid fluidized beds in vortex chambers, Chem. Eng. Process. 85 (2014) 256-290.

[62] A. Gonzalez-Quiroga, P.A. Reyniers, S.R. Kulkarni, M.M. Torregrosa, P. Perreault, G.J. Heynderickx, K.M. Van Geem, G.B. Marin, Design and cold flow testing of a gas-solid vortex reactor demonstration unit for biomass fast pyrolysis, Chem. Eng. J. 329 (2017) 198-210.

[63] R.E. Rosensweig, Fluidization: hydrodynamic stabilization with a magnetic field, Science 204 (1979) 57-60.

[64] J.M. Valverde, M.J. Espin, M.A.S. Quintanilla, A. Castellanos, Fluid to solid transition in magnetofluidized beds of fine powders, J. Appl. Phys. 108 (2010) 054903.

[65] D. Lepek, J.M. Valverde, R. Pfeffer, R.N. Dave, Enhanced nanofluidization by alternating electric fields, AlChE J. 56 (2010) 54-65.

[66] F. Kleijn van Willigen, J.R. van Ommen, J. van Turnhout, C.M. van den Bleek, Bubble size reduction in a fluidized bed by electric fields, Int. J. Chem. React. Eng. 1 (2003) A21.

[67] H. Nakamura, T. Iwasaki, S. Watano, Experimental analysis of bubble velocity in a rotating fluidized bed, Chem. Eng. Process. 48 (2009) 178-186.

[68] J. Quevedo, R. Pfeffer, Y. Shen, R. Dave, H. Nakamura, S. Watano, Fluidization of nanoagglomerates in a rotating fluidized bed, AlChE J. 52 (2006) 2401-2412.

[69] D. Jia, O. Cathary, J. Peng, X. Bi, C.J. Lim, S. Sokhansanj, Y. Liu, R. Wang, A. Tsutsumi, Fluidization and drying of biomass particles in a vibrating fluidized bed with pulsed gas flow, Fuel Process. Technol. 138 (2015) 471-482.

[70] H. Jin, Z. Tong, J. Zhang, B. Zhang, Homogeneous fluidization characteristics of vibrating fluidized beds, Can. J. Chem. Eng. 82 (2004) 1048-1053. 
[71] R. Samson, C.E. Brakel, A.M. Scott, K. Chandrasekharan, P. Veenstra, A bubble model describing the influence of internals on gas fluidization, 10th Int. Symp. on Chem. Rea. Eng., Pergamon, 1988, pp. 2215-2220.

[72] Y. Zhang, J.R. Grace, X. Bi, C. Lu, M. Shi, Effect of louver baffles on hydrodynamics and gas mixing in a fluidized bed of FCC particles, Chem. Eng. Sci. 64 (2009) 3270-3281.

[73] S. Yang, L. Peng, W. Liu, H. Zhao, X. Lv, H. Li, Q. Zhu, Simulation of hydrodynamics in gas-solid bubbling fluidized bed with louver baffles in three dimensions, Powder Technol. 296 (2016) 37-44.

[74] H. Taofeeq, M. Al-Dahhan, Heat transfer and hydrodynamics in a gas-solid fluidized bed with vertical immersed internals, Int. J. Heat Mass Trans. 122 (2018) 229-251.

[75] B.B. Mandelbrot, The fractal geometry of nature, 2nd Ed., W.H. Freeman Company, New York, 1983.

[76] M.-O. Coppens, Method for operating a chemical and/or physical process by means of a hierarchical fluid injection system, US6333019B1 (2001).

[77] M.-O. Coppens, Scaling-up and -down in a nature-inspired way, Ind. Eng. Chem. Res. 44 (2005) 5011-5019.

[78] J. De Wilde, A. de Broqueville, Experimental investigation of a rotating fluidized bed in a static geometry, Powder Technol. 183 (2008) 426-435.

[79] B. Sreenivasan, V.R. Raghavan, Hydrodynamics of a swirling fluidised bed, Chem. Eng. Process. 41 (2002) 99-106.

[80] K. Sirisomboon, P. Laowthong, Experimental investigation and prediction of heat transfer in a swirling fluidized-bed combustor, Appl. Therm. Eng. 147 (2019) 718-727.

[81] W.R. Trujillo, J. De Wilde, Computational fluid dynamics simulation of fluid catalytic cracking in a rotating fluidized bed in a static geometry, Ind. Eng. Chem. Res. 49 (2010) 5288-5298.

[82] J.Z. Kovacevic, M.N. Pantzali, K. Niyogi, N.G. Deen, G.J. Heynderickx, G.B. Marin, Solids velocity fields in a cold-flow gas-solid vortex reactor, Chem. Eng. Sci. 123 (2015) 220-230.

[83] A.C. Louwes, L. Basile, R. Yukananto, J.C. Bhagwandas, E.A. Bramer, G. Brem, Torrefied biomass as feed for fast pyrolysis: An experimental study and chain analysis, Biomass Bioenergy 105 (2017) 116-126.

[84] P. Eliaers, A. de Broqueville, A. Poortinga, T. van Hengstum, J. De Wilde, High-G, low-temperature coating of cohesive particles in a vortex chamber, Powder Technol. 258 (2014) 242-251.

[85] R. Kaewklum, V.I. Kuprianov, Experimental studies on a novel swirling fluidized-bed combustor using an annular spiral air distributor, Fuel 89 (2010) 43-52.

[86] S. Zhu, S.W. Lee, Co-combustion performance of poultry wastes and natural gas in the advanced swirling fluidized bed combustor (SFBC), Waste Manage. 25 (2005) 511-518.

[87] R. Kaewklum, V.I. Kuprianov, P.L. Douglas, Hydrodynamics of air-sand flow in a conical swirling fluidized bed: A comparative study between tangential and axial air entries, Energy Convers. Manage. 50 (2009) 2999-3006. 
[88] C. Sobrino, A. Acosta-Iborra, D. Santana, M. de Vega, Bubble characteristics in a bubbling fluidized bed with a rotating distributor, Int. J. Multiphase Flow 35 (2009) 970-976.

[89] A. Bakhurji, X. Bi, J.R. Grace, Hydrodynamics and solids mixing in fluidized beds with inclined-hole distributors, Particuol. 43 (2019) 19-28.

[90] A. Penn, C.M. Boyce, N. Conzelmann, G. Bezinge, K.P. Pruessmann, C.R. Müller, Real-time magnetic resonance imaging of fluidized beds with internals, Chem. Eng. Sci. 198 (2019) 117-123.

[91] S.E. Lehmann, E.U. Hartge, A. Jongsma, I.M. deLeeuw, F. Innings, S. Heinrich, Fluidization characteristics of cohesive powders in vibrated fluidized bed drying at low vibration frequencies, Powder Technol. 357 (2019) 54-63.

[92] H. Katz, P. Jefferson, Method of stabilizing a fluidized bed using a glow discharge, US3304249A (1965).

[93] J. Hristov, Magnetic field assisted fluidization - a unified approach Part 1. Fundamentals and relevant hydrodynamics of gasfluidized beds (batch solids mode), Rev. Chem. Eng. 18 (2002) 295.

[94] J.M. Valverde, M.J. Espin, M.A.S. Quintanilla, A. Castellanos, Magnetofluidization of fine magnetite powder, Phys. Rev. E 79 (2009) 031306.

[95] M.J. Rhodes, X.S. Wang, A.J. Forsyth, K.S. Gan, S. Phadtajaphan, Use of a magnetic fluidized bed in studying Geldart Group B to A transition, Chem. Eng. Sci. 56 (2001) 5429-5436.

[96] M.J. Espin, J.M. Valverde, M.A.S. Quintanilla, Stabilization of fluidized beds of particles magnetized by an external field: effects of particle size and field orientation, J. Fluid Mech. 732 (2013) 282-303.

[97] M.J. Espin, M.A.S. Quintanilla, J.M. Valverde, Magnetic stabilization of fluidized beds: Effect of magnetic field orientation, Chem. Eng. J. 313 (2017) 1335-1345.

[98] M.J. Espin, M.A.S. Quintanilla, J.M. Valverde, Effect of temperature on flow properties of magnetofluidized beds at low consolidations, Chem. Eng. J. 361 (2019) 50-59.

[99] L. Zhang, Y. He, X. Yang, H. Yuan, Z. Du, Y. Wu, Oxidative carbonylation of phenol to diphenyl carbonate by Pd/MOMnFe2O4 magnetic catalyst, Chem. Eng. J. 278 (2015) 129-133.

[100] B. Zong, X. Meng, X. Mu, X. Zhang, Magnetically stabilized bed reactors, Chinese J. Catal. 34 (2013) 61-68.

[101] A. Tschöpe, M. Wyrwoll, M. Schneider, K. Mandel, M. Franzreb, A magnetically induced fluidized-bed reactor for intensification of electrochemical reactions, Chem. Eng. J. 385 (2020) 123845.

[102] J. Zhang, M. Zhang, W. Li, X. Li, X. Meng, B. Zong, Local heat transfer properties in co- and counter-current G-L-S magnetically stabilized fluidized beds, Particuol. 9 (2011) 44-50.

[103] C.V. Whittman, B.O. Ademoyega, Hydrodynamic changes and chemical reaction in a transparent two-dimensional crossflow electrofluidized bed. 1. Experimental results, Ind. Eng. Chem. Res. 26 (1987) 1586-1593.

[104] F. Kleijn van Willigen, J.R. van Ommen, J. van Turnhout, C.M. van den Bleek, Bubble size reduction in electric-fieldenhanced fluidized beds, J. Electrostat 63 (2005) 943-948. 
[105] Y. Yang, S. Ge, Y. Zhou, J. Sun, Z. Huang, J. Wang, M. Lungu, Z. Liao, B. Jiang, Y. Yang, Effects of DC electric fields on meso-scale structures in electrostatic gas-solid fluidized beds, Chem. Eng. J. 332 (2018) 293-302.

[106] Q. Yu, R.N. Dave, C. Zhu, J.A. Quevedo, R. Pfeffer, Enhanced fluidization of nanoparticles in an oscillating magnetic field, AlChE J. 51 (2005) 1971-1979.

[107] F. Karimi, M. Haghshenasfard, R. Sotudeh-Gharebagh, R. Zarghami, N. Mostoufi, Multiscale characterization of nanoparticles in a magnetically assisted fluidized bed, Particuol. 51 (2020) 64-71.

[108] F.J. Higuera, Analysis of electrodynamic fluidization, J. Fluid Mech. 854 (2018) 261-292.

[109] R. Rakoczy, A. Przybył, M. Kordas, M. Konopacki, R. Drozd, K. Fijałkowski, The study of influence of a rotating magnetic field on mixing efficiency, Chem. Eng. Process. 112 (2017) 1-8.

[110] C. Reichhardt, C.J.O. Reichhardt, Controlled fluidization, mobility, and clogging in obstacle arrays using periodic perturbations, Phys. Rev. Lett. 121 (2018) 068001.

[111] S. Kaart, Controlling chaotic bubbles, Doctoral thesis in Chemical Engineering, Delft University of Technology, 2002.

[112] S. Kaart, J.C. Schouten, C.M. van den Bleek, Improving conversion and selectivity of catalytic reactions in bubbling gassolid fluidized bed reactors by control of the nonlinear bubble dynamics, Catal. Today 48 (1999) 185-194.

[113] L. Massimilla, G. Volpicelli, G. Raso, A study on pulsing gas fluidization of beds of particles, AIChE Symp. Ser. 62 (1966) $63-70$.

[114] P. Russo, R. Chirone, L. Massimilla, S. Russo, The influence of the frequency of acoustic waves on sound-assisted fluidization of beds of fine particles, Powder Technol. 82 (1995) 219-230.

[115] F. Raganati, V. Gargiulo, P. Ammendola, M. Alfe, R. Chirone, CO2 capture performance of HKUST-1 in a sound assisted fluidized bed, Chem. Eng. J. 239 (2014) 75-86.

[116] F. Raganati, P. Ammendola, R. Chirone, Effect of acoustic field on CO2 desorption in a fluidized bed of fine activated carbon, Particuol. 23 (2015) 8-15.

[117] J.R. van Ommen, J.M. Valverde, R. Pfeffer, Fluidization of nanopowders: a review, J. Nanopart. Res. 14 (2012) 737.

[118] K. Noda, Y. Mawatari, S. Uchida, Flow patterns of fine particles in a vibrated fluidized bed under atmospheric or reduced pressure, Powder Technol. 99 (1998) 11-14.

[119] E. Ireland, K. Pitt, R. Smith, A review of pulsed flow fluidisation; the effects of intermittent gas flow on fluidised gas-solid bed behaviour, Powder Technol. 292 (2016) 108-121.

[120] Y. Mawatari, T. Koide, Y. Tatemoto, T. Takeshita, K. Noda, Comparison of three vibrational modes (twist, vertical and horizontal) for fluidization of fine particles, Adv. Powder Technol. 12 (2001) 157-168.

[121] E. Cano-Pleite, F. Hernández-Jiménez, M. de Vega, A. Acosta-Iborra, Experimental study on the motion of isolated bubbles in a vertically vibrated fluidized bed, Chem. Eng. J. 255 (2014) 114-125. 
[122] C. Zeilstra, M.A. van der Hoef, J.A.M. Kuipers, Experimental and numerical study of solids circulation in gas-vibro fluidized beds, Powder Technol. 248 (2013) 153-160.

[123] T. Zhou, H. Kage, H. Li, Bubble characteristics in a two-dimensional vertically vibro-fluidized bed, Particuol. 3 (2005) 224228.

[124] Y. Zhang, J. Zhang, Y. Zhao, X. Zhang, X. Yang, E. Zhou, C. Duan, G. Wang, L. Dong, Investigations on dynamics of bubble in a 2D vibrated fluidized bed using pressure drop signal and high-speed image analysis, Chem. Eng. J. 395 (2020) 125129.

[125] P. Zhao, Y. Zhao, Z. Chen, Z. Luo, Dry cleaning of fine lignite in a vibrated gas-fluidized bed: Segregation characteristics, Fuel 142 (2015) 274-282.

[126] C.H. Nam, R. Pfeffer, R.N. Dave, S. Sundaresan, Aerated vibrofluidization of silica nanoparticles, AlChE J. 50 (2004) $1776-1785$.

[127] E. Marco, A. Santos, M. Menéndez, J. Santamaría, Fluidization of agglomerating particles: influence of the gas temperature and composition on the fluidization of a Li/MgO catalyst, Powder Technol. 92 (1997) 47-52.

[128] M. Saidi, H. Basirat Tabrizi, J.R. Grace, A review on pulsed flow in gas-solid fluidized beds and spouted beds: Recent work and future outlook, Adv. Powder Technol. 30 (2019) 1121-1130.

[129] M. Kobayashi, D. Ramaswami, W. Brazelton, Pulsed-bed approach to fluidization, Chem. Eng. Progr., Symp. Ser. 66 (1970) 47-57.

[130] H. Wong, M. Baird, Fluidisation in a pulsed gas flow, Chem. Eng. J. 2 (1971) 104-113.

[131] A. Akhavan, F. Rahman, S. Wang, M. Rhodes, Enhanced fluidization of nanoparticles with gas phase pulsation assistance, Powder Technol. 284 (2015) 521-529.

[132] N.A. Moussa, A.A. Fowle, Exploratory study of pulsed atmospheric fluidized bed combustion, in: Proc. of 8th Int. Conf. on FCB, 1985, pp. 1300-1310.

[133] Y. Li, F. Zhu, Y. Zhang, Y. Zhao, G. Zhang, Q. Huang, L. Dong, Characterization of bubble behaviors in a dense phase pulsed gas-solid fluidized bed for dry coal processing, Particuol. (2020).

[134] H. Khosravi Bizhaem, H. Basirat Tabrizi, Experimental study on hydrodynamic characteristics of gas--solid pulsed fluidized bed, Powder Technol. 237 (2013) 14-23.

[135] J. Werther, Influence of the bed diameter on the hydrodynamics of gas fluidized beds, AIChE Symp. Ser. 70 (1974) 53-62.

[136] S. Mori, C.Y. Wen, Estimation of bubble diameter in gaseous fluidized beds, AlChE J. 21 (1975) 109-115.

[137] D. Jia, X. Bi, C.J. Lim, S. Sokhansanj, A. Tsutsumi, Biomass drying in a pulsed fluidized bed without inert bed particles, Fuel 186 (2016) 270-284.

[138] Y. Liu, H. Ohara, A. Tsutsumi, Pulsation-assisted fluidized bed for the fluidization of easily agglomerated particles with wide size distributions, Powder Technol. 316 (2017) 388-399. 
[139] L. Shen, F. Johnsson, B. Leckner, Digital image analysis of hydrodynamics two-dimensional bubbling fluidized beds, Chem. Eng. Sci. 59 (2004) 2607-2617.

[140] J.F. Davidson, D. Harrison, J.R.F.G.D. Carvalho, On the liquidlike behavior of fluidized beds, Annu. Rev. Fluid Mech. 9 (1977) 55-86.

[141] L. Dong, Y. Zhao, C. Duan, Z. Luo, B. Zhang, X. Yang, Characteristics of bubble and fine coal separation using active pulsing air dense medium fluidized bed, Powder Technol. 257 (2014) 40-46.

[142] M. Saidi, H. Basirat Tabrizi, S. Chaichi, M. Dehghani, Pulsating flow effect on the segregation of binary particles in a gassolid fluidized bed, Powder Technol. 264 (2014) 570-576.

[143] K. Zhang, S. Wang, Y. Tang, Y. He, Prediction of segregation behavior of binary mixture in a pulsed fluidized bed, Adv. Powder Technol. 30 (2019) 2659-2665.

[144] L.J.J. Lim, E.W.C. Lim, Mixing and segregation behaviors of a binary mixture in a pulsating fluidized bed, Powder Technol. 345 (2019) 311-328.

[145] L. Dong, Y. Zhang, Y. Zhao, L. Peng, E. Zhou, L. Cai, B. Zhang, C. Duan, Effect of active pulsing air flow on gas-vibro fluidized bed for fine coal separation, Adv. Powder Technol. 27 (2016) 2257-2264.

[146] L. Dong, B. Zhang, Y. Zhang, Y. Zhao, E. Zhou, P. Lv, C. Duan, Kinetic characteristics of the particles in a dense-phase pulsed fluidized bed for dry beneficiation, Can. J. Chem. Eng. 95 (2017) 1133-1140.

[147] M. Köksal, H. Vural, Bubble size control in a two-dimensional fluidized bed using a moving double plate distributor, Powder Technol. 95 (1998) 205-213.

[148] I.A. Bokun, S.S. Zabrodskii, Experimental study of the heat transfer between a pulsating bed and the heating surface, Nauk. Tekh. (1966) 27-32.

[149] D.V. Pence, D.E. Beasley, Heat transfer in pulse-stabilized fluidization - Part 1: overall cylinder and average local analyses, Int. J. Heat Mass Trans. 45 (2002) 3609-3619.

[150] D. Zhang, M. Koksal, Heat transfer in a pulsed bubbling fluidized bed, Powder Technol. 168 (2006) 21-31.

[151] K. Zhang, S. Wang, B. Li, Y. He, Y. Zhao, Heat transfer in a pulsed fluidized bed by using coupled CFD-DEM method, Powder Technol. 367 (2020) 497-505.

[152] J. Blacha-Jurkiewicz, Z. Gawrzynski, R. Glaser, Drying of granular material in pulsofluidized bed, Drying 87 (1987) 109114.

[153] A. Akhavan, J.R. van Ommen, J. Nijenhuis, X.S. Wang, M.-O. Coppens, M.J. Rhodes, Improved drying in a pulsationassisted fluidized bed, Ind. Eng. Chem. Res. 48 (2008) 302-309.

[154] D. Jia, X. Bi, C.J. Lim, S. Sokhansanj, A. Tsutsumi, Heat transfer in a tapered fluidized bed of biomass particles with pulsed gas flow, Particuol. 42 (2019) 2-14. 
[155] J.F. Kok, E.J.R. Parteli, T.I. Michaels, D.B. Karam, The physics of wind-blown sand and dust, Rep. Prog. Phys. 75 (2012) 106901.

[156] G.E. Liston, M. Sturm, A snow-transport model for complex terrain, J. Glaciol. 44 (2017) 498-516.

[157] M.O. Green, G. Coco, Review of wave-driven sediment resuspension and transport in estuaries, Rev. Geophys. 52 (2014) $77-117$

[158] L.P. Kadanoff, Built upon sand: Theoretical ideas inspired by granular flows, Rev. Mod. Phys. 71 (1999) $435-444$.

[159] M.C. Cross, P.C. Hohenberg, Pattern formation outside of equilibrium, Rev. Mod. Phys. 65 (1993) 851.

[160] B. Hao, H.T. Bi, Forced bed mass oscillations in gas-solid fluidized beds, Powder Technol. 149 (2005) 51-60.

[161] S. Douady, S. Fauve, C. Laroche, Subharmonic instabilities and defects in a granular layer under vertical vibrations, Europhys. Lett. 8 (1989) 621.

[162] F. Melo, P.B. Umbanhowar, H.L. Swinney, Transition to parametric wave patterns in a vertically oscillated granular layer, Phys. Rev. Lett. 72 (1994) 172.

[163] F. Melo, P.B. Umbanhowar, H.L. Swinney, Hexagons, kinks, and disorder in oscillated granular layers, Phys. Rev. Lett. 75 (1995) 3838 .

[164] M. Faraday, On a peculiar class of acoustical figures; and on certain forms assumed by groups of particles upon vibrating elastic surfaces, Philos. Trans. R. Soc. London 121 (1831) 299-340.

[165] W. Zhang, J. Vinals, Pattern formation in weakly damped parametric surface waves driven by two frequency components, J. Fluid Mech. 341 (1997) 225-244.

[166] P.B. Umbanhowar, F. Melo, H.L. Swinney, Localized excitations in a vertically vibrated granular layer, Nature 382 (1996) 793-796.

[167] S.J. Moon, M.D. Shattuck, C. Bizon, D.I. Goldman, J.B. Swift, H.L. Swinney, Phase bubbles and spatiotemporal chaos in granular patterns, Phys. Rev. E 65 (2001) 011301.

[168] O. Sano, Density wave as a mechanism of the formation of ripples in vertically oscillated thicker granular layer, J. Phys. Soc. Jpn. 80 (2011) 034402.

[169] S. Luding, E. Clément, J. Rajchenbach, J. Duran, Simulations of pattern formation in vibrated granular media, Europhys. Lett. 36 (1996) 247.

[170] T.H. Metcalf, J.B. Knight, H.M. Jaeger, Standing wave patterns in shallow beds of vibrated granular material, Phys. A 236 (1997) 202-210.

[171] E. Clément, L. Labous, Pattern formation in a vibrated granular layer, Granular Gases (2001) 233-243.

[172] P.B. Umbanhowar, H.L. Swinney, Wavelength scaling and square/stripe and grain mobility transitions in vertically oscillated granular layers, Phys. A 288 (2000) 344-362. 
[173] N. Mujica, F. Melo, Experimental study of solid-liquid-type transitions in vibrated granular layers and the relation with surface waves, Phys. Rev. E 63 (2000) 011303.

[174] P.B. Umbanhowar, F. Melo, H.L. Swinney, Periodic, aperiodic, and transient patterns in vibrated granular layers, Phys. A 249 (1998) 1-9.

[175] D.I. Goldman, M.D. Shattuck, S.J. Moon, J.B. Swift, H.L. Swinney, Lattice dynamics and melting of a nonequilibrium pattern, Phys. Rev. Lett. 90 (2003) 104302.

[176] N. Mujica, F. Melo, Solid-liquid transition and hydrodynamic surface waves in vibrated granular layers, Phys. Rev. Lett. 80 (1998) 5121.

[177] K.M. Aoki, T. Akiyama, Spontaneous wave pattern formation in vibrated granular materials, Phys. Rev. Lett. 77 (1996) 4166.

[178] C. Bizon, M.D. Shattuck, J.R. de Bruyn, J.B. Swift, W.D. McCormick, H.L. Swinney, Convection and diffusion in patterns in oscillated granular media, J. Stat. Phys. 93 (1998) 449-465.

[179] C. Bizon, M.D. Shattuck, J.B. Swift, W.D. McCormick, H.L. Swinney, Patterns in 3D vertically oscillated granular layers: simulation and experiment, Phys. Rev. Lett. 80 (1998) 57.

[180] J. Bougie, J. Kreft, J.B. Swift, H.L. Swinney, Onset of patterns in an oscillated granular layer: Continuum and molecular dynamics simulations, Phys. Rev. E 71 (2005) 021301.

[181] H.K. Park, H.T. Moon, Square to stripe transition and superlattice patterns in vertically oscillated granular layers, Phys. Rev. E 65 (2002) 051310.

[182] A. Goldshtein, M. Shapiro, Mechanics of collisional motion of granular materials. Part 1. General hydrodynamic equations, J. Fluid Mech. 282 (2006) 75-114.

[183] J.T. Jenkins, M.W. Richman, Kinetic theory for plane flows of a dense gas of identical, rough, inelastic, circular disks, Phys. Fluids 28 (1985) 3485-3494.

[184] E. Cerda, F. Melo, S. Rica, Model for subharmonic waves in granular materials, Phys. Rev. Lett. 79 (1997) 4570.

[185] J. Bougie, K. Duckert, Continuum simulations of shocks and patterns in vertically oscillated granular layers, Phys. Rev. E 83 (2011) 011303.

[186] M.-O. Coppens, M.A. Regelink, C.M. van den Bleek, Pulsation induced transition from chaos to periodically ordered patterns in fluidised beds, in: Proc. of 4th World Conf. on Part. Technol. (WCPT), 2002, pp. 1-8.

[187] J. Li, I. Aranson, W.-K. Kwok, L. Tsimring, Periodic and disordered structures in a modulated gas-driven granular layer, Phys. Rev. Lett. 90 (2003) 134301.

[188] L. de Martín, C. Ottevanger, J.R. van Ommen, M.-O. Coppens, Universal stability curve for pattern formation in pulsed gassolid fluidized beds of sandlike particles, Phys. Rev. Fluids 3 (2018) 034303. 
[189] A.P. Baskakov, V.G. Tuponogov, N.F. Filippovsky, A study of pressure fluctuations in a bubbling fluidized bed, Powder Technol. 45 (1986) 113-117.

[190] L. de Martín, Influence of particle dynamics on the instability for pattern formation in shallow pulsed beds, Physical Review Fluids 3 (2018) 124304.

[191] J.C. Schouten, C.M. van den Bleek, Chaotic hydrodynamics of fluidization: consequences for scaling and modeling of fluid bed reactors, AlChE Symp. Ser. 88 (1992) 70-70.

[192] C. Goniva, C. Kloss, N.G. Deen, J.A.M. Kuipers, S. Pirker, Influence of rolling friction on single spout fluidized bed simulation, Particuol. 10 (2012) 582-591.

[193] M. Syamlal, W. Rogers, T.J. O’Brien, MFIX documentation: Theory guide, National Energy Technology Laboratory, Technical Note DOE/METC-95/1013 and NTIS/DE95000031, 1993.

[194] Z. Luo, M. Fan, Y. Zhao, X. Tao, Q. Chen, Z. Chen, Density-dependent separation of dry fine coal in a vibrated fluidized bed, Powder Technol. 187 (2008) 119-123.

[195] M.A.A. Cruz, M.L. Passos, W.R. Ferreira, Final drying of whole milk powder in vibrated-fluidized beds, Drying Technol. 23 (2005) 2021-2037.

[196] E. Marring, A.C. Hoffmann, L.P.B.M. Janssen, The effect of vibration on the fluidization behaviour of some cohesive powders, Powder Technol. 79 (1994) 1-10.

[197] H.T. Bi, A critical review of the complex pressure fluctuation phenomenon in gas-solids fluidized beds, Chem. Eng. Sci. 62 (2007) 3473-3493.

[198] V. Francia, K. Wu, M.-O. Coppens, Self-organization of gas bubbles in frictional granular media: oscillating fluidized beds, (2020) (In preparation).

[199] K. Wu, V. Francia, M.-O. Coppens, Dynamic viscoplastic granular flows: A persistent challenge in gas-solid fluidization, Powder Technol. 365 (2020) 172-185.

[200] A.M. Turing, The chemical basis of morphogenesis, Philos. Trans. R. Soc. London. Ser. B 237 (1952) $37-72$.

[201] L.A. Vandewalle, V. Francia, K.M. van Geem, G.B. Marin, M.-O. Coppens, Dynamically structured gas-solid fluidized beds: lateral mixing and compartmentalization, (2020) (In preparation).

[202] V. Verma, J.T. Padding, N.G. Deen, J.A.M. Kuipers, F. Barthel, M. Bieberle, M. Wagner, U. Hampel, Bubble dynamics in a 3-D gas-solid fluidized bed using ultrafast electron beam X-ray tomography and two-fluid model, AlChE J. 60 (2014) $1632-1644$. [203] J. Saayman, W. Nicol, J.R. van Ommen, R.F. Mudde, Fast X-ray tomography for the quantification of the bubbling-, turbulent- and fast fluidization-flow regimes and void structures, Chem. Eng. J. 234 (2013) 437-447.

[204] Y.T. Makkawi, P.C. Wright, Fluidization regimes in a conventional fluidized bed characterized by means of electrical capacitance tomography, Chem. Eng. Sci. 57 (2002) 2411-2437. 
[205] Y.T. Makkawi, P.C. Wright, Electrical capacitance tomography for conventional fluidized bed measurements—remarks on the measuring technique, Powder Technol. 148 (2004) 142-157.

[206] J.N. Sines, S. Hwang, Q.M. Marashdeh, A. Tong, D. Wang, P. He, B.J. Straiton, C.E. Zuccarelli, L.-S. Fan, Slurry bubble column measurements using advanced electrical capacitance volume tomography sensors, Powder Technol. 355 (2019) 474-480. [207] C.M. Boyce, N.P. Rice, A. Ozel, J.F. Davidson, A.J. Sederman, L.F. Gladden, S. Sundaresan, J.S. Dennis, D.J. Holland, Magnetic resonance characterization of coupled gas and particle dynamics in a bubbling fluidized bed, Phys. Rev. Fluids 1 (2016) 074201.

[208] A. Penn, C.M. Boyce, T. Kovar, T. Tsuji, K.P. Pruessmann, C.R. Müller, Real-time magnetic resonance imaging of bubble behavior and particle velocity in fluidized beds, Ind. Eng. Chem. Res. 57 (2018) 9674-9682.

[209] M. Rüdisüli, T.J. Schildhauer, S.M.A. Biollaz, J.R. van Ommen, Bubble characterization in a fluidized bed by means of optical probes, Int. J. Multiphase Flow 41 (2012) 56-67.

[210] C. Rautenbach, R.F. Mudde, X. Yang, M.C. Melaaen, B.M. Halvorsen, A comparative study between electrical capacitance tomography and time-resolved X-ray tomography, Flow Meas. Instrum. 30 (2013) 34-44.

[211] A. Bakshi, M. Shahnam, A. Gel, T. Li, C. Altantzis, W. Rogers, A.F. Ghoniem, Comprehensive multivariate sensitivity analysis of CFD-DEM simulations: Critical model parameters and their impact on fluidization hydrodynamics, Powder Technol. (2018).

[212] J.E. Higham, M. Shahnam, A. Vaidheeswaran, On the dynamics of a quasi-two-dimensional pulsed-fludized bed, arXiv preprint arXiv:1809.05033 (2018).

[213] N. Deen, M. van Sint Annaland, M. van der Hoef, J.A.M. Kuipers, Review of discrete particle modeling of fluidized beds, Chem. Eng. Sci. 62 (2007) 28-44.

[214] M. van der Hoef, M. van Sint Annaland, N.G. Deen, J.A.M. Kuipers, Numerical simulation of dense gas-solid fluidized beds: A multiscale modeling strategy, Annu. Rev. Fluid Mech. 40 (2008) 47-70.

[215] R. Jackson, The dynamics of fluidized particles, Cambridge University Press, Cambridge, 2000.

[216] D. Gidaspow, Multiphase flow and fluidization: continuum and kinetic theory descriptions, Academic press, New York, 1994.

[217] Y. Tsuji, T. Kawaguchi, T. Tanaka, Discrete particle simulation of two-dimensional fluidized bed, Powder Technol. 77 (1993) 79-87.

[218] R. van de Klundert, Pattern formation in pulsated fludized beds and vertically vibrated granular layers, MSc Thesis in Chemical Engineering, Delft University of Technology, 2001.

[219] T.E. Broadhurst, Particle oscillation in fluidized beds, Encyclopedia of Fluid Mechanics 4 (1986) 781-815.

[220] T. Kawaguchi, A. Miyoshi, T. Tanaka, Y. Tsuji, Discrete particle analysis of 2D pulsating fluidized bed, in: Proc. of 4th Int. Conf. on Multiphase Flow, 2001, pp. 838. 
[221] X. Wang, M. Rhodes, Pulsed fluidization—a DEM study of a fascinating phenomenon, Powder Technol. 159 (2005) 142149.

[222] S. Sundaresan, Instabilities in fluidized beds, Annu. Rev. Fluid Mech. 35 (2003) 63-88.

[223] K. Wu, L. de Martín, L. Mazzei, M.-O. Coppens, Pattern formation in fluidized beds as a tool for model validation: A twofluid model based study, Powder Technol. 295 (2016) 35-42.

[224] A. Bakshi, C. Altantzis, A. Bershanska, A.K. Stark, A.F. Ghoniem, On the limitations of 2D CFD for thin-rectangular fluidized bed simulations, Powder Technol. 332 (2018) 114-119.

[225] D.G. de Oliveira, C.L. Wu, K. Nandakumar, Numerical investigation of pulsed fluidized bed using CFD-DEM: Insights on the dynamics, Powder Technol. 363 (2020) 745-756.

[226] S. Chialvo, S. Sundaresan, A modified kinetic theory for frictional granular flows in dense and dilute regimes, Phys. Fluids 25 (2013) 070603.

[227] Y.T. Makkawi, R. Ocone, Modelling the Particle Stress at the Dilute-intermediate-dense Flow Regimes: A Review, KONA Powder Part. J. 23 (2005) 49-63.

[228] B. van Wachem, J.C. Schouten, C.M. van den Bleek, R. Krishna, J. Sinclair, Comparative analysis of CFD models of dense gas-solid systems, AlChE J. 47 (2001) 1035-1051.

[229] P.C. Johnson, P. Nott, R. Jackson, Frictional-collisional equations of motion for participate flows and their application to chutes, J. Fluid Mech. 210 (1990) 501-535.

[230] R. Ocone, S. Sundaresan, R. Jackson, Gas-particle flow in a duct of arbitrary inclination with particle-particle interactions, AlChE J. 39 (1993) 1261-1271.

[231] D.G. Schaeffer, Instability in the evolution equations describing incompressible granular flow, J. Differ. Equations 66 (1987) $19-50$.

[232] L. Mazzei, P. Lettieri, A drag force closure for uniformly dispersed fluidized suspensions, Chem. Eng. Sci. 62 (2007) 61296142.

[233] M. van der Hoef, M. Ye, M. van Sint Annaland, A. Andrews, S. Sundaresan, J.A.M. Kuipers, Multiscale modeling of gasfluidized beds, Adv. Chem. Eng. 31 (2006) 65-149.

[234] J.T. Jenkins, C. Zhang, Kinetic theory for identical, frictional, nearly elastic spheres, Phys. Fluids 14 (2002) $1228-1235$.

[235] J. Sun, F. Battaglia, Hydrodynamic modeling of particle rotation for segregation in bubbling gas-fluidized beds, Chem. Eng. Sci. 61 (2006) 1470-1479.

[236] J.T. Jenkins, D. Berzi, Dense inclined flows of inelastic spheres: tests of an extension of kinetic theory, Granular Matter 12 (2010) 151-158.

[237] J. Li, W. Huang, From Multiscale to Mesoscience: Addressing Mesoscales in Mesoregimes of Different Levels, Annual Review of Chemical and Biomolecular Engineering 9 (2018) 41-60. 
[238] J. Li, W. Huang, J. Chen, W. Ge, C. Hou, Mesoscience based on the EMMS principle of compromise in competition, Chem. Eng. J. 333 (2018) 327-335.

[239] L. Yang, J.T. Padding, J.A.M. Kuipers, Modification of kinetic theory of granular flow for frictional spheres, part II: Model validation, Chem. Eng. Sci. 152 (2016) 783-794.

[240] L. Yang, J.T. Padding, J.A.M. Kuipers, Modification of kinetic theory of granular flow for frictional spheres, Part I: Twofluid model derivation and numerical implementation, Chem. Eng. Sci. 152 (2016) 767-782.

[241] G.D.R. MiDi, On dense granular flows, Eur. Phys. J. E 14 (2004) 341-365.

[242] P. Jop, Y. Forterre, O. Pouliquen, A constitutive law for dense granular flows, Nature 441 (2006) 727-730.

[243] S. Chialvo, J. Sun, S. Sundaresan, Bridging the rheology of granular flows in three regimes, Phys. Rev. E 85 (2012) 021305.

[244] J. Sun, S. Sundaresan, A constitutive model with microstructure evolution for flow of rate-independent granular materials, J. Fluid Mech. 682 (2011) 590-616.

[245] K. Kamrin, G. Koval, Nonlocal constitutive relation for steady granular flow, Phys. Rev. Lett. 108 (2012) 178301.

[246] K. Kamrin, D.L. Henann, Nonlocal modeling of granular flows down inclines, Soft Matter 11 (2015) 179-185.

[247] D.I. Goldman, J.B. Swift, H.L. Swinney, Noise, coherent fluctuations, and the onset of order in an oscillated granular fluid, Phys. Rev. Lett. 92 (2004) 174302. 